\title{
FLOW PHENOMENA IN THE VERY NEAR WAKE OF A FLAT PLATE WITH A CIRCULAR TRAILING EDGE
}

\author{
Man Mohan Rai ${ }^{1}$ \\ NASA Ames Research Center, Moffett Field, CA-94035
}

\begin{abstract}
The very near wake of a flat plate with a circular trailing edge, exhibiting pronounced shedding of wake vortices, is investigated with data from a direct numerical simulation. The separating boundary layers are turbulent and statistically identical thus resulting in a wake that is symmetric in the mean. The focus here is on the instability of the detached shear layers, the evolution of ribvortex induced localized regions of reverse flow that detach from the main body of reverse flow in the trailing edge region and convect downstream, and phaseaveraged velocity statistics in the very near wake. The detached shear layers are found to exhibit unstable behavior intermittently, including the development of shear layer vortices as in earlier cylinder flow investigations with laminar separating boundary layers. Only a small fraction of the separated turbulent boundary layers undergo this instability, and form the initial shed vortices. Pressure spectra within the shear layers show a broadband peak at a multiple of shedding frequency. Phase-averaged intensity and shear stress distributions of the randomly fluctuating component of velocity are compared with those obtained in the near wake. The distributions of the production terms in the transport equations for the turbulent stresses are also provided.
\end{abstract}

\section{INTRODUCTION}

A deep understanding of wakes with shed vortices is critical to many engineering design processes. In turbomachines, for example, the near wake is of interest in trailing edge design, and the intermediate wake is of interest in understanding and predicting flow transition and unsteady loads on downstream airfoils. Turbulence models and design criteria are a couple of areas that would benefit from a better comprehension of wakes. Cylinder wakes have been studied extensively to gain a better understanding of the basic flow phenomena that are encountered in such flows. These investigations focus on the low Reynolds number range where the cylinder boundary layer is laminar. However, from an engineering point of view, there is considerable interest in the situation where the upper and/or lower boundary layer of an airfoil is turbulent, and these turbulent boundary layers separate from the airfoil to contribute to the formation of the wake downstream.

Experimental, computational and theoretical means have been employed in efforts to study wakes (Refs. 1 \& 2 and the references cited therein provide an extensive review). While much has been accomplished there are many important issues that need to be resolved. The physics of the very near wake (less than three trailing edge diameters downstream) is perhaps the most challenging of them all. This region comprises the two detached shear layers, the recirculation region and wake flow. The interaction amongst these three components is to some extent still a matter of conjecture. Experimental techniques have generated a large percentage of the cylinder data that have provided us with the current state of understanding of the subject. More recently computational techniques have been used to simulate cylinder wakes, and the data from such simulations are being used to both refine our understanding of such flows as well as provide new insights.

\footnotetext{
${ }^{1}$ Senior Scientist for Computational Sciences, Exploration Technology Directorate. Associate Fellow, AIAA.
} 
Here we investigate the wake of a flat plate with turbulent boundary layers that exhibits pronounced vortex shedding. Unlike the cylinder, and more in keeping with the airfoil, the boundary layers are turbulent even though the Reynolds number based on the trailing edge diameter $\left(R e_{D}, D\right.$ is the diameter) is modest $(10,000)$. With the flat plate there is the advantage of not having the complexities of the airfoil (such as pressure gradients and specificity of the geometry). In this investigation we utilize direct numerical simulation (DNS) data obtained with a high-order accurate, upwind-biased finitedifference method. The upper and lower surface boundary layers are both turbulent and statistically identical. Thus the computed wake is symmetric in a statistical sense. Results from the DNS including flow visualization and turbulence statistics (phase-averaged) in the near wake $(x / D>3.0)$ were presented in Ref. 1. The focus in the present study is on the very near wake $(x / D<3.0)$. The goals are to investigate the stability of the turbulent detached shear layer, rib vortex induced regions of separation that are detached from the main body of reverse flow near the trailing edge, and the distribution of the phaseaveraged velocity statistics in the very near wake. The stability of the laminar separating shear layer has been investigated in depth in Ref. 2 and the references cited therein. The intermittent generation of shear layer vortices, the mechanisms that initiate these vortices and aid in their growth, a possible classification of the different types of instability and other items of interest are discussed in Ref. 2. Here we look for shear layer vortices in the case of the turbulent separating shear layer. Once again we are interested in the instability mechanisms, the evolution of shear layer vortices in time, a characteristic frequency that is most probably a function of $\mathrm{Re}_{\mathrm{D}}$, and, the roll-up of the shear layers that results in the shed vortices.

\section{COMPUTATIONAL GRID AND NUMERICAL METHOD}

The computational region is divided into two zones to facilitate grid generation and provide adequate grid resolution for the wake. Figure 1 shows the plate cross-section and the two zones that comprise the computational region. The three-dimensional zones and grids are obtained by uniformly spacing copies of these two-dimensional zones in the spanwise direction (z). The plate zone is bounded by four boundaries: the plate surface (excluding the trailing edge), an external boundary and, two zonal boundaries (top and bottom) that interface with the wake zone. It captures the inviscid flow-field upstream of the trailing edge and the plate boundary layers. The leading edge of the plate is an ellipse. The wake zone is constructed to provide adequate grid resolution for the detached shear layers, the recirculation region and the wake. The boundaries of this zone include the circular trailing edge, the upper and lower boundaries and the exit boundary. Both the upper and lower boundaries consist of a zonal boundary segment that interfaces with the plate zone, and a second segment that serves as an external boundary.

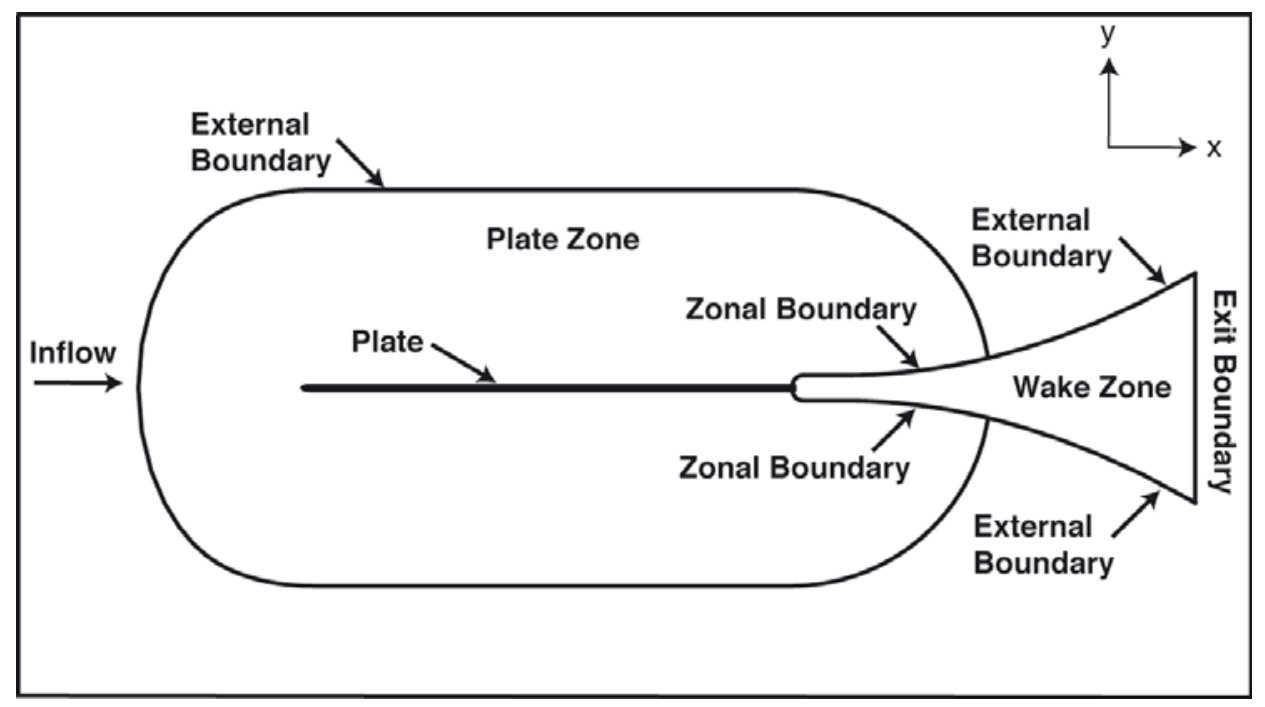

Figure 1. Midspan plate section and multiple zone discretization of the computational region (from Ref.1). 
The external boundary of the plate zone is placed $50.0 \mathrm{D}$ from the plate surface, where $\mathrm{D}$ is the diameter of the circular trailing edge ( $D$ is also the thickness of the plate). The length of the plate is 125.5D. The exit boundary of the wake zone is $104.0 \mathrm{D}$ from the center of the circular trailing edge (coordinate system origin) and, the vertical extent of this boundary is $81.0 \mathrm{D}$. The vertical extent of the wake zone near the trailing edge, where its upper/lower boundaries are horizontal, is $9.0 \mathrm{D}$ and is large enough to completely contain the wake. The spanwise extent of the region is 4.0D. The streamwise extent of the well-resolved region of the wake extends to 13.87D from the center of the circular trailing edge.

Figure 2 shows representative grids in the vicinity of the trailing edge in both zones. These grids were generated with an algebraic grid generator. Both the grids have the same spacing in the wall normal direction at the plate surface, $\Delta n / D=0.002$. The grid in the wake zone transitions from curvilinear near the trailing edge to rectangular downstream. Beyond 13.87D, the wake grid coarsens gradually in the streamwise direction. In addition to reducing the computational costs incurred, this coarsening dissipates the wake to a degree that inviscid exit boundary conditions can be employed at the exit boundary of the wake zone. The wake grid was constructed with 741 grid points in the streamwise direction, 411 in the cross-stream direction and 256 in the spanwise direction (about $78 \times 10^{6}$ grid points). The wake grid used in Ref. 1 consisted of about $43 \times 10^{6}$ grid points. The additional grid points in the present simulation were used primarily to provide more resolution for the detached shear layers and are thus, for the most part, concentrated in the first 5.0D downstream of the trailing edge. The resolution achieved along the centerline in the three spatial directions at $x / D=10.0$ is approximately $\Delta x / \eta=3.7, \Delta y / \eta=2.2$ and $\Delta z / \eta=$ 2.1 where $\eta$ is the computed Kolmogorov length scale at the same location. The grid spacing in the $y$ direction increases gradually from the centerline to the upper/lower wake zone boundaries to $\Delta y / \eta=4.0$. In comparison the resolution achieved in these directions in Ref. 1 at $x / D=10.0, y / D=0.0$, is approximately $\Delta x / \eta=4.0, \Delta y / \eta=4.0$ and $\Delta z / \eta=2.10$; the stretching in the $y$ direction is minor. The present grid is better or equally well resolved in all directions. The number of grid points used in the plate zone is identical to that in Ref. 1 and the resolution obtained in each direction is discussed in detail there.

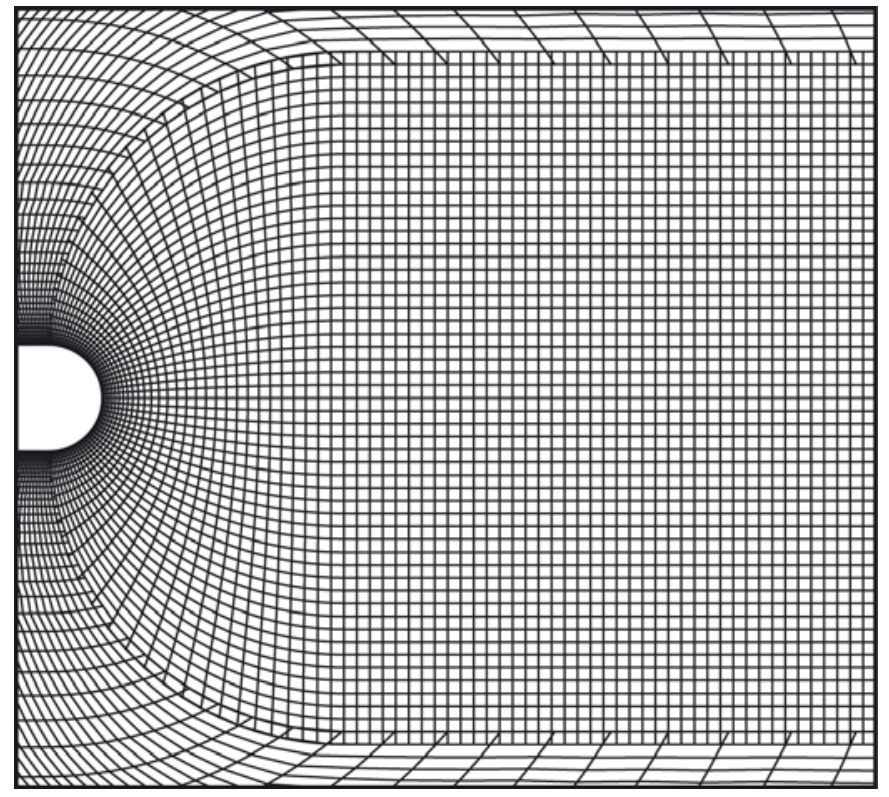

Figure 2. Representative grids in the plate and wake zones in the trailing edge region (from Ref. 1).

The high-order accurate upwind-biased method developed in Refs. $3 \& 4$ is used here to compute the flow over the plate as well as that in the wake. The inviscid terms are computed using sixth- and seventh-order upwind-biased finite differences, both with seventh-order dissipation terms. The viscous terms are computed with fourth-order central differences. As discussed above the computational region is 
discretized using the plate grid and the wake grid. The boundaries that contain these grids can be broadly classified as natural and zonal boundaries. The natural boundaries include the external boundary of the plate grid, the surface of the plate, the exit boundary of the wake grid, the segments of the upper and lower boundaries of the wake grid labeled as "external boundary" in Fig. 1, and, the boundaries in the spanwise direction. The upstream segment of the upper boundary between the plate and wake grids is an example of the zonal boundaries used in the computation. Both the natural and zonal boundary conditions used in this study are discussed in Ref. 3. Periodic boundary conditions are imposed on the boundaries in the spanwise direction (homogeneity in z). No-slip/adiabatic wall conditions are used on the plate surface and the trailing edge circle. Wall blowing/suction, as described in Ref. 3, is implemented on a short segment on both the upper and lower surfaces of the plate to induce transition to turbulence. The boundary layer is fully turbulent upstream of the trailing edge (some details are provided in the next section). The upper and lower transitional/turbulent boundary layers and the wake are computed via DNS.

\section{RESULTS}

Both the boundary layers and the wake were computed via DNS. The computations were performed at a Mach number of 0.2. The Reynolds numbers based on the diameter and plate length were 10,000 and $1,255,000$, respectively. Data sampling was initiated after the initial transients were eliminated. Turbulence statistics were obtained via averaging in the spanwise direction and in time or at constant phase as appropriate. The sampling period consisted of 175,000 time steps (approximately 100 shedding periods with 1750 time steps per period). The computed Strouhal number was 0.173 .

\section{The Plate Section}

Here, as in Ref. 1, we establish that the plate boundary layer is turbulent before it detaches from the plate surface. Figure 3 shows the mean velocity profile in the turbulent region at $90 \%$ of the length of the plate (12.55D upstream of the circular trailing edge). The dashed lines represent the near wall behavior and the log-law; $\mathrm{u}^{+}$is the streamwise component of velocity normalized by the wall shear velocity and $\mathrm{y}^{+}$is the wall normal distance in wall coordinates. The agreement with the linear profile and the log-law is good. The computed normal turbulent intensity profiles are compared with the experimental data of Ref. 5 in Ref. 1 and are in good agreement. The average percentage difference in rms turbulence intensity (u \& v components) at $\operatorname{Re}_{\theta} \sim 2400$ (Reynolds number based on momentum thickness) is $6.7 \%$. The average percentage difference in $w_{r m s}$ at $R_{\theta} \sim 1750$ is $4.3 \%$.

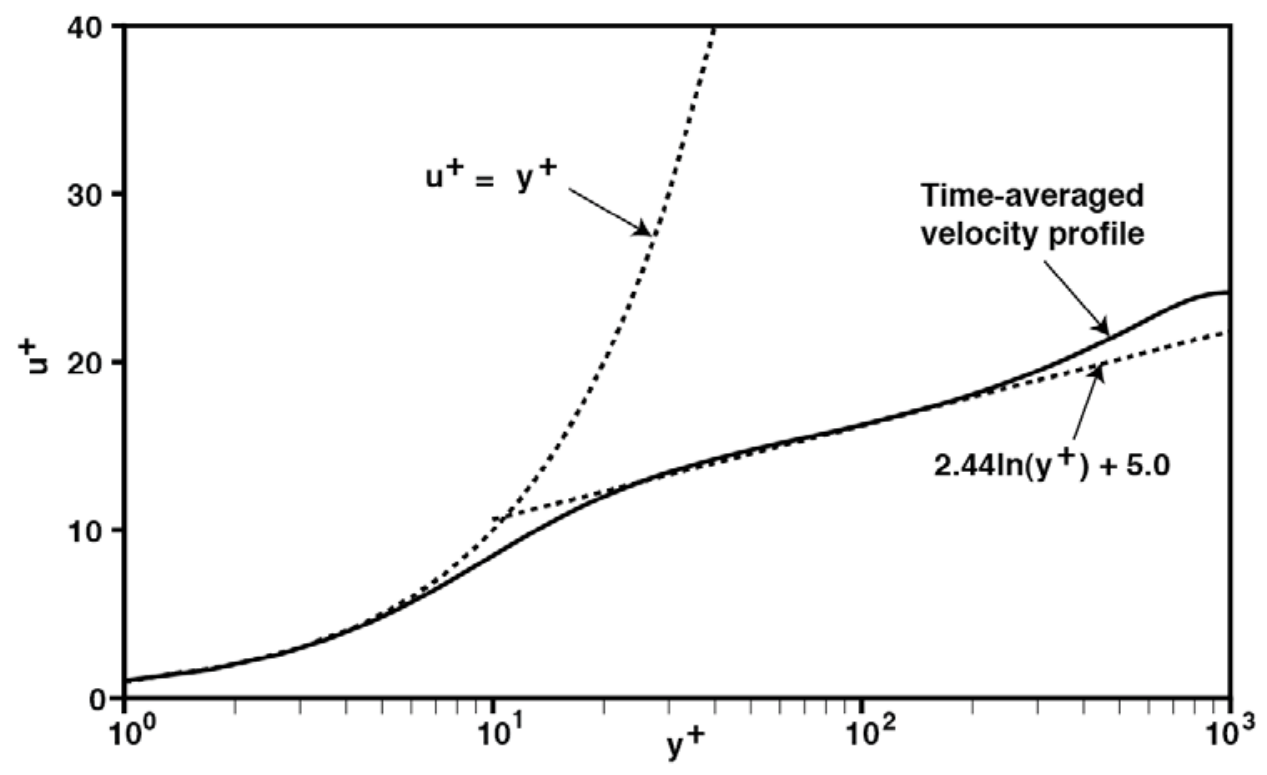

Figure 3 . Mean velocity profile at $90 \%$ of plate length. 
The intensity profiles computed here are nearly identical to those of Ref. 1 at these two values of $\operatorname{Re}_{\theta}$. Here and in the rest of the article $u, v \& w$ are the velocities in the $x, y \& z$ directions respectively, and $p$ is the pressure. The shape factor variation on the plate surface showed a rapid reduction through transition followed by a nearly constant region thereafter except near the trailing edge; it is approximately 1.40 at $90 \%$ of the plate length. Thus, the flow at this location closely approximates a turbulent boundary layer.

\section{Detached Shear Layer Instability}

A fairly extensive review of the instability of the detached shear layer in the case of the cylinder with laminar separating boundary layers is provided in Ref. 2 . Measurements of fluctuating velocity in the vicinity of such shear layers first led to the discovery ${ }^{6}$ of 'transition waves'. They were determined to be of higher frequency than the large-scale vortex shedding frequency and result in a broadband peak in velocity spectra obtained near the shear layer. The ratio of the shear-layer frequency $\left(\omega_{\mathrm{sl}}\right.$, defined as the frequency at which the spectrum peaks in this broadband region) to the shedding frequency $\left(\omega_{\text {st }}\right)$ was initially determined to be proportional to the square root of the Reynolds number $\left(\operatorname{Re}_{\mathrm{D}}\right)$ in Ref. 6 ; the exponent has been more accurately estimated to be around $2 / 3$ in subsequent efforts. Additional details can be found in Ref. 2 and the references cited therein.

The transition waves of Ref. 6 occur intermittently and often start as small-amplitude disturbances in the shear layer that grow in magnitude and in some cases eventually become a stream of shear-layer vortices that can be easily visualized via contours of spanwise vorticity. In Ref. 2 it was determined that a principal cause for both the initiation and growth of this instability is an interaction between the shear layer and vortices found in the recirculation region. The interaction results in an undulation of the shear-layer that in turn results in both initiation and growth of the instability. A classification of this instability and the differences in causative effects from class to class is provided in Ref. 2.

The computational dataset of the current investigation presents an excellent opportunity to determine if shear layer vortices are generated when the separating boundary layers are turbulent. If so, there are many questions to be answered. Is the phenomenon intermittent? Do spectra of various fluid mechanical quantities obtained in the shear layers exhibit a broadband peak as in the earlier cylinder cases? How does the ratio $\omega_{\mathrm{Sl}} / \omega_{\mathrm{st}}$ vary with $\mathrm{Re}_{\mathrm{D}}$ ? What is the principal cause of the instability? Do recirculation region vortices play an important role in the initiation and growth of shear layer vortices? What role do the turbulent fluctuations in the separating boundary layer play in this process (absent in the cylinder cases)? Are the shear layer vortices localized in the spanwise direction? Does the shear layer essentially break down during particularly powerful shear-layer vortex generation periods (labeled as Class $A_{1}$ in the cylinder case)? We provide the answers to some of these questions in this section.

The data provided in the next few figures were obtained during the data-sampling period (after the initial transients were eliminated). The time-step $n=0$ corresponds to the time at which sampling was initiated. In the following contour plots (except pressure) the colors blue/green represent negative values (deep blue representing the lowest value) of the term/quantity being discussed. Orange, red and magenta represent positive values (magenta bordering on white representing the highest value). Shades of yellow represent values close to zero.

Figure 4 shows instantaneous spanwise vorticity contours in an $(x, y)$ plane at $z / D=1.125$ during the seventh shedding period (at $n=10760$ and $T / T_{p}=6.15$, where $T_{p}$ is the shedding period). The upper and lower detached shear layers are evident (lower one marked as DSL). The turbulent boundary layer is much thicker than the region depicted by the closely spaced contours corresponding to the shear layers. The vertical bar near the upper left-hand corner of the figure is $\theta / 2$ in length. Here $\theta$ is the momentum thickness at about $94 \%$ plate length. The momentum thickness peaks at this location before decreasing slightly in the trailing edge region because of a favorable pressure gradient. The remarkable feature of the rollup of the shear layers, that results in the initial formation of the shed vortices, is that only a very small 
fraction of the turbulent boundary layer participates in this process. Here onward the expression 'detached shear layer' (or DSL) denotes only that fraction of the separated boundary layer seen in Fig. 4.

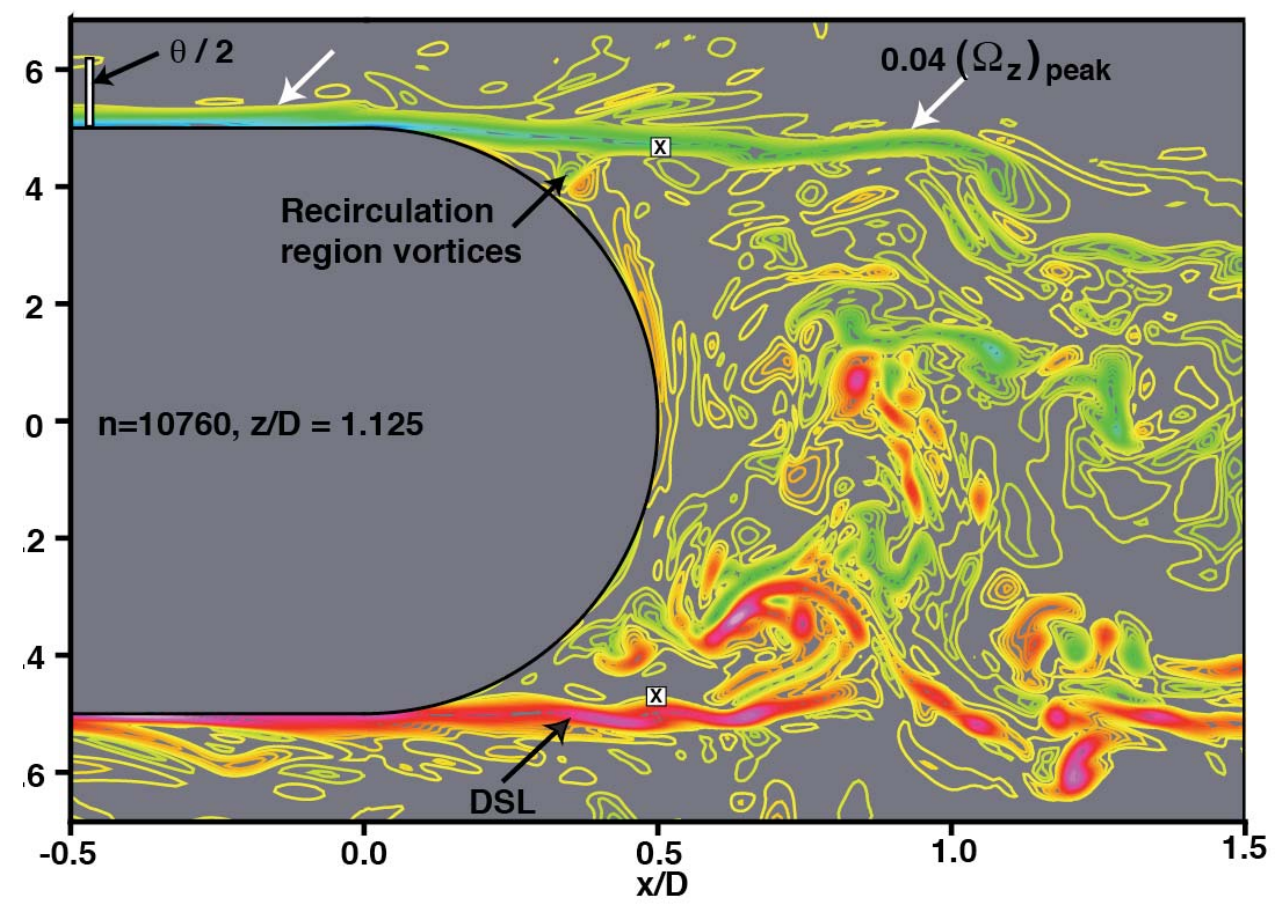

Figure 4. Instantaneous spanwise vorticity contours during the seventh shedding period $\left(T / T_{p}=6.15\right)$; $\mathrm{red} / \mathrm{magenta}$ represent high/highest positive values, green/blue low/lowest negative values, yellow $\approx 0.0$.

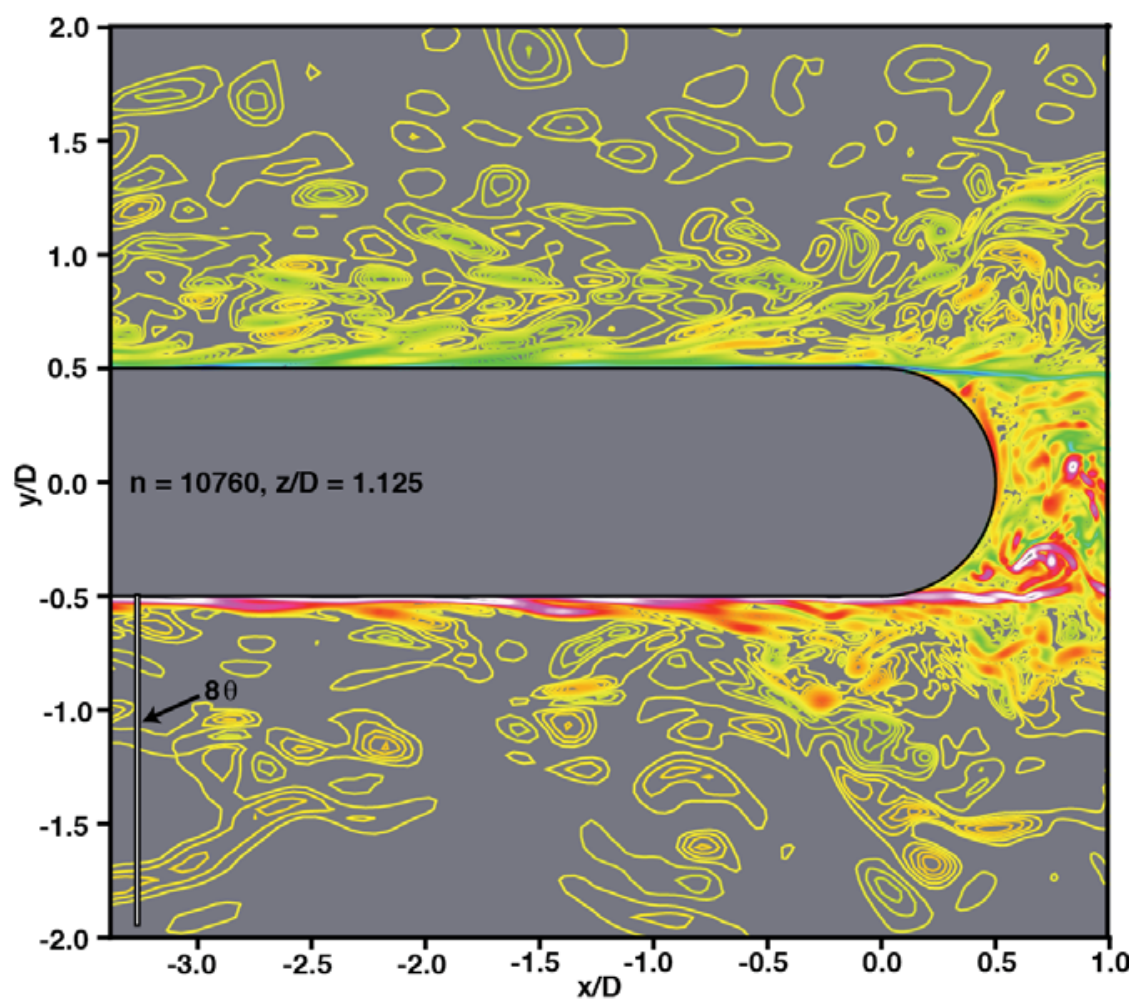

Figure 5. Instantaneous spanwise vorticity contours at $\mathrm{T} / \mathrm{T}_{\mathrm{p}}=6.15$ (expanded view); $\mathrm{red} / \mathrm{magenta}$ represent high/highest positive values, green/blue low/lowest negative values, yellow $\approx 0.0$. 
Some quantification of this definition of the DSL is desirable. Time-averaged spanwise vorticity contours show a peak in magnitude immediately downstream of $x / D=0.0$ on the trailing edge surface. The outer contour line both in the region of the boundary layer and the upper DSL (highlighted with white arrows) corresponds to about $4 \%$ of this peak value. The thickness of the DSL, as defined by this magnitude of vorticity, varies between $\theta / 4$ and $\theta / 6$ in Fig. 4.

Figure 5 shows instantaneous spanwise vorticity contours at the same instant as in Fig. 4 but over a larger region that includes the upstream turbulent boundary layers. The minimum and maximum values of vorticity are half as much as in Fig. 4 and the number of contour levels has been doubled, effectively reducing the increments in contour levels by a factor of 4 . This is necessary to visualize the weaker turbulent eddies in the log-layer of the boundary layer. The vertical bar is about $8 \theta$ in length and thus represents a substantial portion of the boundary layer thickness. This figure demonstrates that only a very small portion of the boundary layer is involved in the initial roll-up into shed vortices. Animations show that eddies in the log-layer convect past the trailing edge with relatively little change.

Unlike in the present case a substantial portion of the boundary layer participates in the roll-up process in the flow past a cylinder with laminar separating boundary layers (data from DNS at $\operatorname{Re}_{\mathrm{D}}=$ $3900^{4}$ ). Figure 6 shows the time-averaged spanwise vorticity contours obtained. The white arrow points to the contour that is $4 \%$ of the peak vorticity level and the black arrow points to a line segment that is $8 \theta$ in length. The detached shear layer contains most of the laminar boundary layer instead of a small fraction.

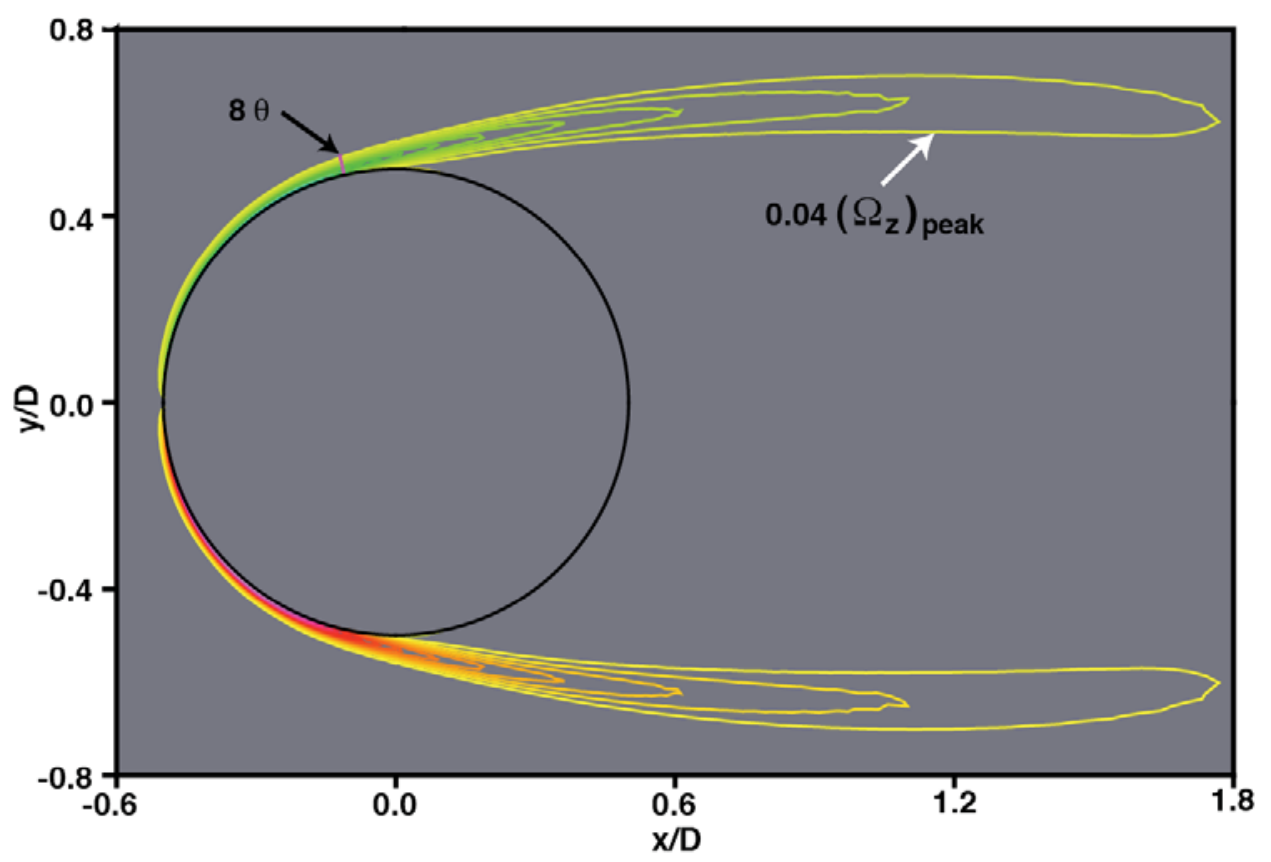

Fig. 6. Time-averaged spanwise vorticity contours for a cylinder at $\operatorname{Re}_{\mathrm{D}}=3900$; $\mathrm{red} / \mathrm{magenta}$ represent high/highest positive values, green/blue low/lowest negative values, yellow $\approx 0.0$.

Figure 4 shows the upper DSL in a relatively quiescent state but interacting with recirculation region vortices. The lower DSL is in the process of rolling up and contains within it a few relatively weak shear layer votices. The disturbances external to the two DSLs $(y / D>0.5)$ are also evident in this figure. The points marked with an ' $x$ ' at $x / D=0.5$ are locations at which the time-varying flow variables are stored and will be analyzed. Figure 7 shows instantaneous spanwise vorticity contours a few time steps later at $T / T_{p}=6.35(n=11,110)$. The two shear layer vortices $(A \& B)$ in the upper shear layer are evident. Clearly shear layer vortices are generated even in this case of the flat plate where the separating boundary layers are turbulent. They scale on the thickness of the DSL (DSL as defined earlier) and not 
the boundary layer thickness. Presumably they are caused by an interaction between the DSL and the recirculation region vortices seen here and in Fig. 4 (this point will be discussed shortly).

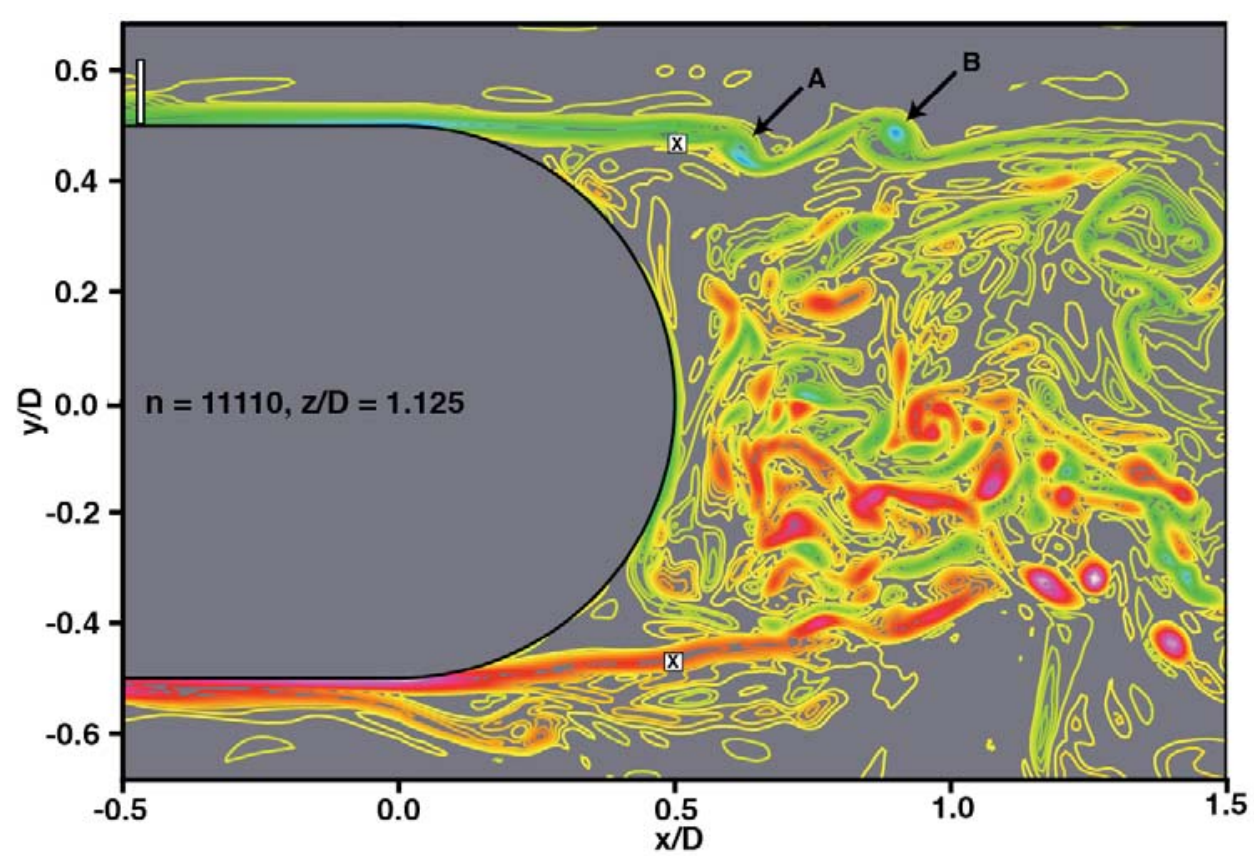

Figure 7. Instantaneous spanwise vorticity contours at $\mathrm{T} / \mathrm{T}_{\mathrm{p}}=6.35$;

$\mathrm{red} /$ magenta represent high/highest positive values, green/blue low/lowest negative values, yellow $\approx 0.0$.

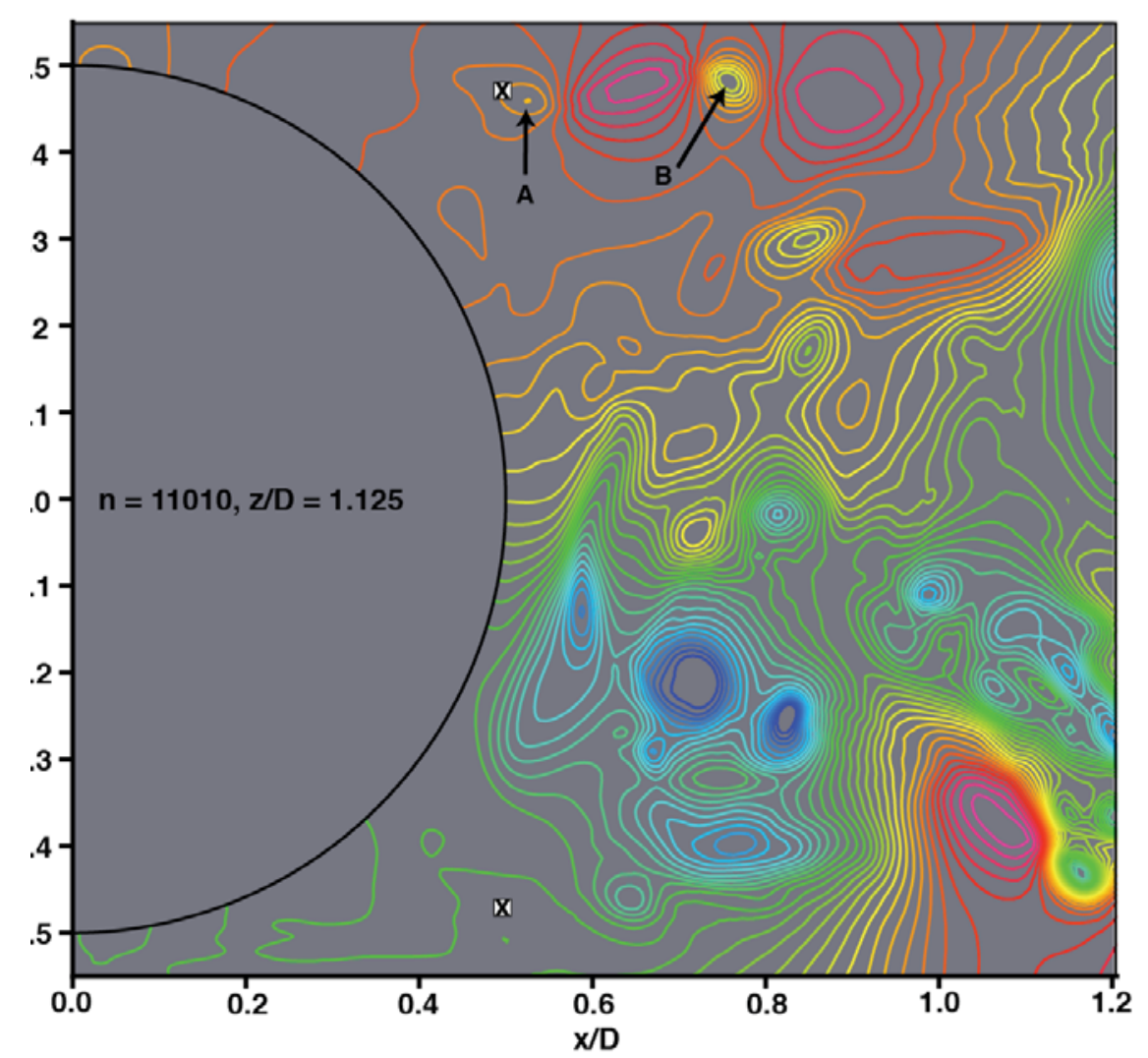

Figure 8. Instantaneous pressure contours at $\mathrm{T} / \mathrm{T}_{\mathrm{p}}=6.29$. 
The computed pressure and velocity components at the points marked with an ' $x$ ' in Figs. $4 \& 7$ were stored during the sampling period. The conventional practice in earlier investigations of shear-layer intermittency has been to compute velocity spectra in the vicinity of the shear layers. Here the incoming velocity fluctuations from the upstream boundary layer are large and thus mask the broadband peak caused by shear layer vortex generation. However, the pressure fluctuations that are a result of the shear layer vortices are sufficiently large in comparison to those in the boundary layer and hence can be used to characterize the range of frequencies that comprise the broadband peak and thus also $\omega_{\mathrm{sl}}$. Figure 8 shows instantaneous pressure contours at a time instant between those of Figs. $4 \& 7, T / T_{p}=6.29$. In this figure vortex $B$ has passed the location ' $x$ ' and convected downstream but vortex $A$ is still in the process of passing over ' $x$ '. The passage of shear layer vortices over a given point creates a pressure minimum in the pressure history that can be used determine $\omega_{\mathrm{sl}}$ via spectral analysis and for other purposes as will be seen shortly. The much more powerful pressure minima in the lower half of Fig. 8 constitute the shed vortex that is still in the formative stage. The shed vortex is not a single cylindrical vortex as in low Reynolds number laminar shedding but is an amalgam of shear layer vortices, recirculation region vortices and segments of the shear layer that wrap around the shed vortex (see also Figs. 4 \& 7). The shed vortex is comprised of smaller vortices of the same sign as the vorticity in the shear layer that is in the process of rolling up and those of opposite sign, with those of the same sign dominating (net value). The shed vortex retains this particular characteristic for several diameters downstream (Ref. 1) and presumably even until it begins disintegrating in the intermediate wake region.

In Ref. 2 contours of velocity fluctuations at points just above the shear layers (the equivalent of the points marked ' $x$ ' here) were plotted in the (t, $z$ ) plane (Fig. 17 in Ref. 2). These contours showed the evolution of each intermittency event (initiation, growth \& decay) and also the spanwise extent of the disturbance as a function of time. This contour plot helped in instability classification and in obtaining parameters of interest within each event such as the rate of generation of shear layer vortices via the monitoring of zero-crossings of the velocity signal. The velocity signal used to generate this plot in Ref. 2 was obtained from a high pass filter applied to the original computed velocity signal in order to eliminate the effect of periodic shedding. Figure 9 shows the application of the same technique to the pressure signals obtained at the ' $x$ ' locations in the upper and lower shear layers. The time period portrayed is eight cycles long. The entire spanwise extent of the computational region (4.0D) is used to obtain this plot. The cut-off frequency for the high-pass filter was set at $1.5 \omega_{\mathrm{st}}$. The plot obtained without the filter showed vertical bands of contours that appeared in a nearly periodic manner in addition to the ones seen in Fig. 9.

Figure 9 shows numerous intermittency events in both the upper and lower shear layers; quiescent periods are also observed in both layers. Each event consists of a few or several vertical bands, each in the blue/green range or red/orange range. The former set of bands is a result of the pressure minima caused by the passing shear layer vortices. The latter set represents a recovery of the pressure that occurs between subsequent shear layer vortices. These appear as maxima in pressure in Fig. 8 (for example, the maximum between vortices A and B). As expected the red \& blue bands appear alternately. The spanwise extent of these bands varies between $0.1 \mathrm{D} \& 0.8 \mathrm{D}$. Thus the intermittency events, as in the case of the cylinder with laminar separating boundary layers ${ }^{2}$, are localized in the $z$ direction and in general, their spanwise extent first increases, reaches a maximum and then decreases.

The intermittency events in the two layers are for the most past uncorrelated. The event investigated in Figs. $4 \& 7$ is marked as ' $A$ ' with an arrow. Often the end of one intermittency period nearly coincides with the beginning of another making it somewhat difficult to ascertain the causative agent for a particular event. However, there are a few events that are preceded by a quiescent period that can be examined for possible causative agents. These events are marked with an ' $x$ ' in Fig. 9. Instantaneous spanwise vorticity contours as in Figs. $4 \& 7$ at these locations showed recirculation region vortices interacting with the DSL, thus indicating that these vortices do indeed instigate the destabilization of the DSL and the generation of shear layer vortices just as in the case of the cylinder with laminar separating boundary layers ${ }^{2}$. Additionally the points marked with an 'o' in Fig. 9 (located in the quiescent regions) all showed little or no interaction of recirculation region vortices with the DSL upstream of the location ' $x$ '. 

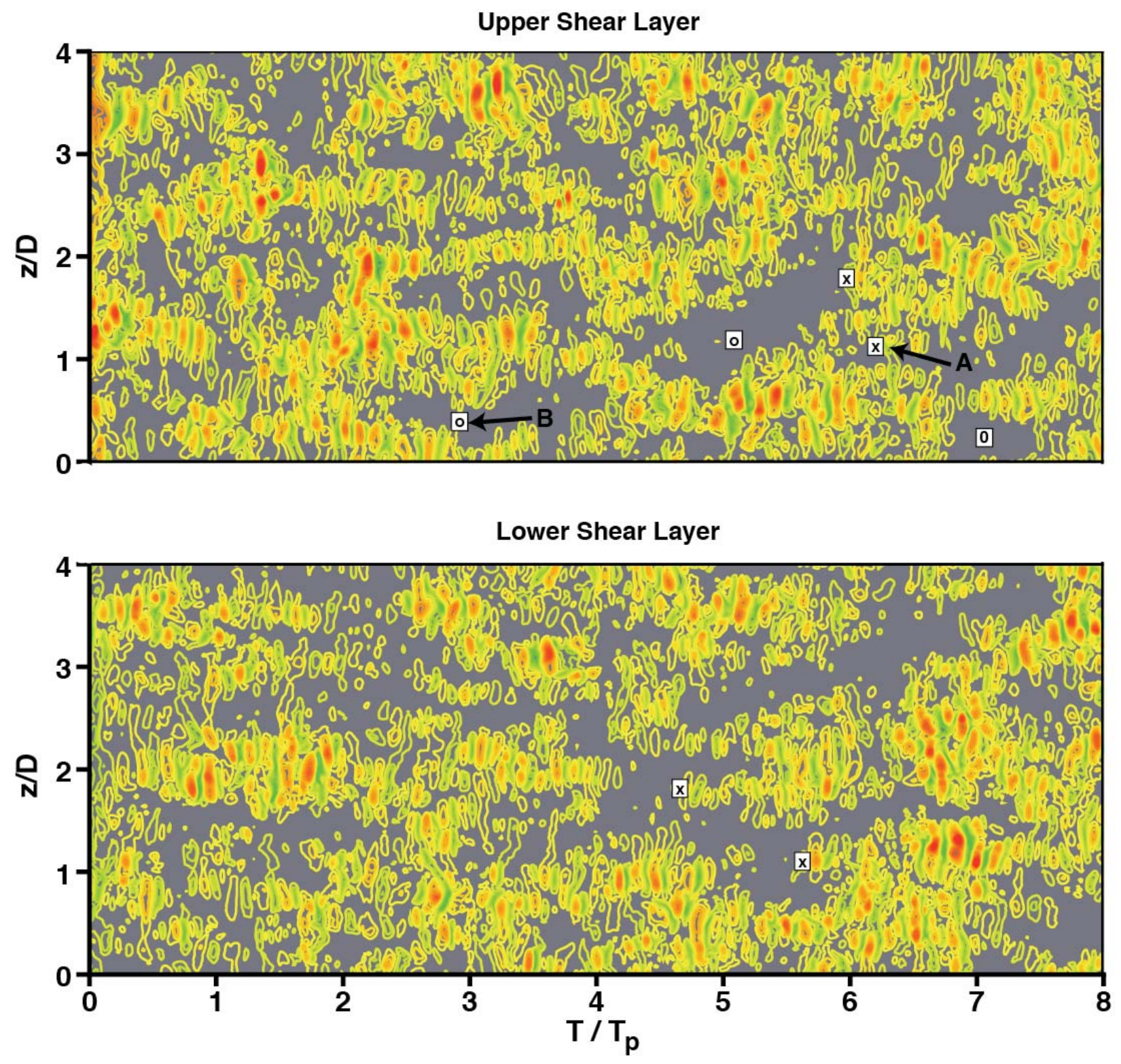

Figure 9. Instantaneous contours of filtered fluctuating pressure in a $(t, z)$ plane at the points marked ' $x$ ' in both shear layers over a period of 8 shedding periods;

$\mathrm{red} / \mathrm{magenta}$ represent high/highest positive values, green/blue low/lowest negative values, yellow $\approx 0.0$.

Instantaneous spanwise vorticity contours at the location marked 'B' in the upper DSL in Fig. 9 are provided in Figure 10. The quiescent nature of the upper DSL and the lack of interaction between this DSL and recirculation region vortices are evident (in the region marked by the arrow). We note here that interactions which occur further downstream of the location ' $x$ ' leave little or no trace at this location. The lack of interactions at all the quiescent points ('o') is further evidence that the interaction discussed above is a primary cause of shear layer instability. The role of disturbances external to the shear layers (on the side away from the centerline) will be discussed in a separate article.

Figure 11 shows the fluctuating pressure signal $\left(p^{\prime}\right)$ over the first eight shedding periods at $z / D=$ 1.125 (high-pass filter applied as in Fig. 9); it includes the intermittency event of Figs. 4 \& 7, also marked ' $A$ ' in Fig. 9. This plot shows about half a dozen intermittency events during this period. Pressure fluctuations during an event are considerably larger than the turbulent fluctuations between events 
(originally from the turbulent boundary layer). Clearly peak fluctuation levels in different events vary considerably (by a factor of two or three). The relatively moderate variation in the widths of the vertical bands in Fig. 9 and the intermittency related fluctuations in Fig. 11 hint at the possibility of a central frequency $\left(\omega_{\mathrm{sl}}\right)$ about which the fluctuating energy is distributed in a broadband manner. In general the events show an initial increase in fluctuating amplitude followed by a decrease later in the event; this is a feature that is related to the increase /decrease seen in the widths of the bands in the $z$ direction in Fig. 9.

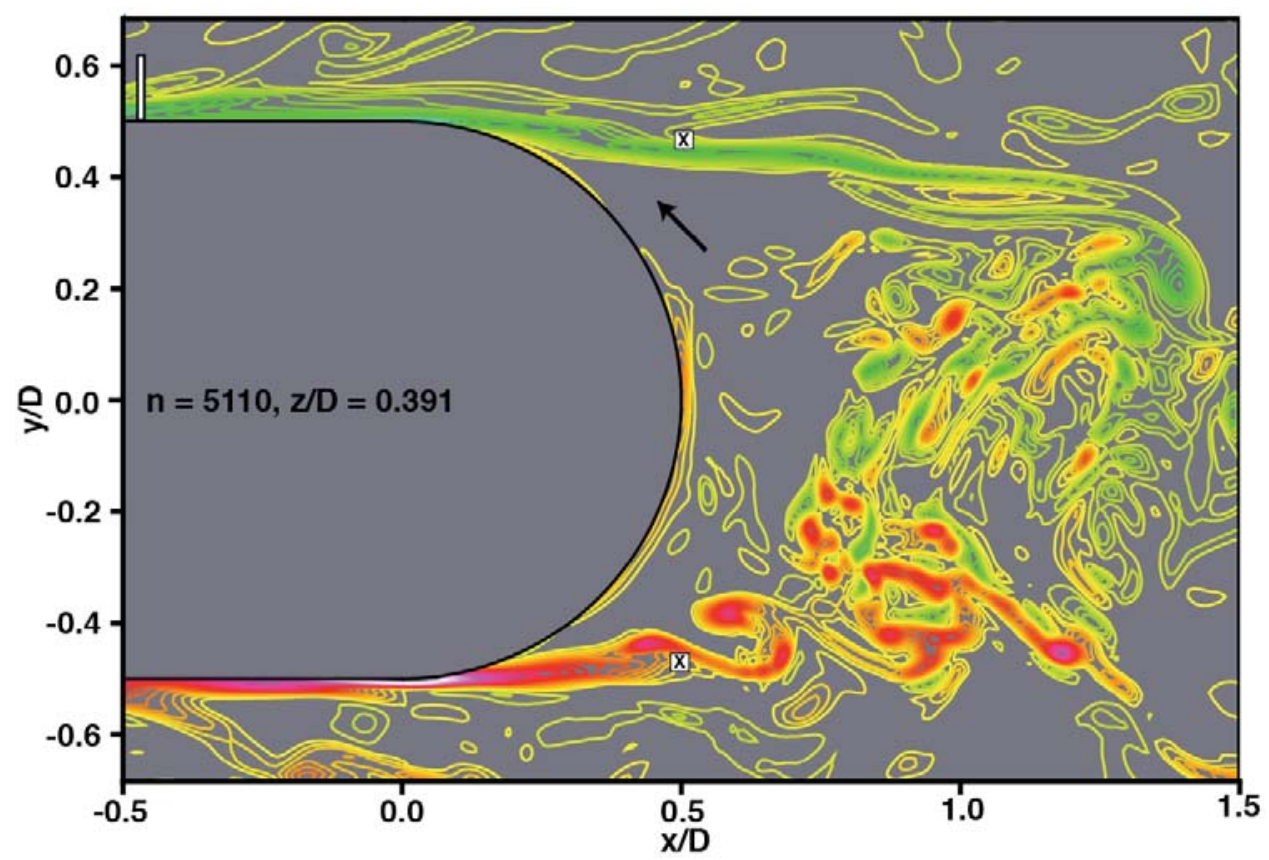

Figure 10. Instantaneous spanwise vorticity contours at $\mathrm{T} / \mathrm{T}_{\mathrm{p}}=2.92$;

$\mathrm{red} / \mathrm{magenta}$ represent high/highest positive values, green/blue low/lowest negative values, yellow $\approx 0.0$.

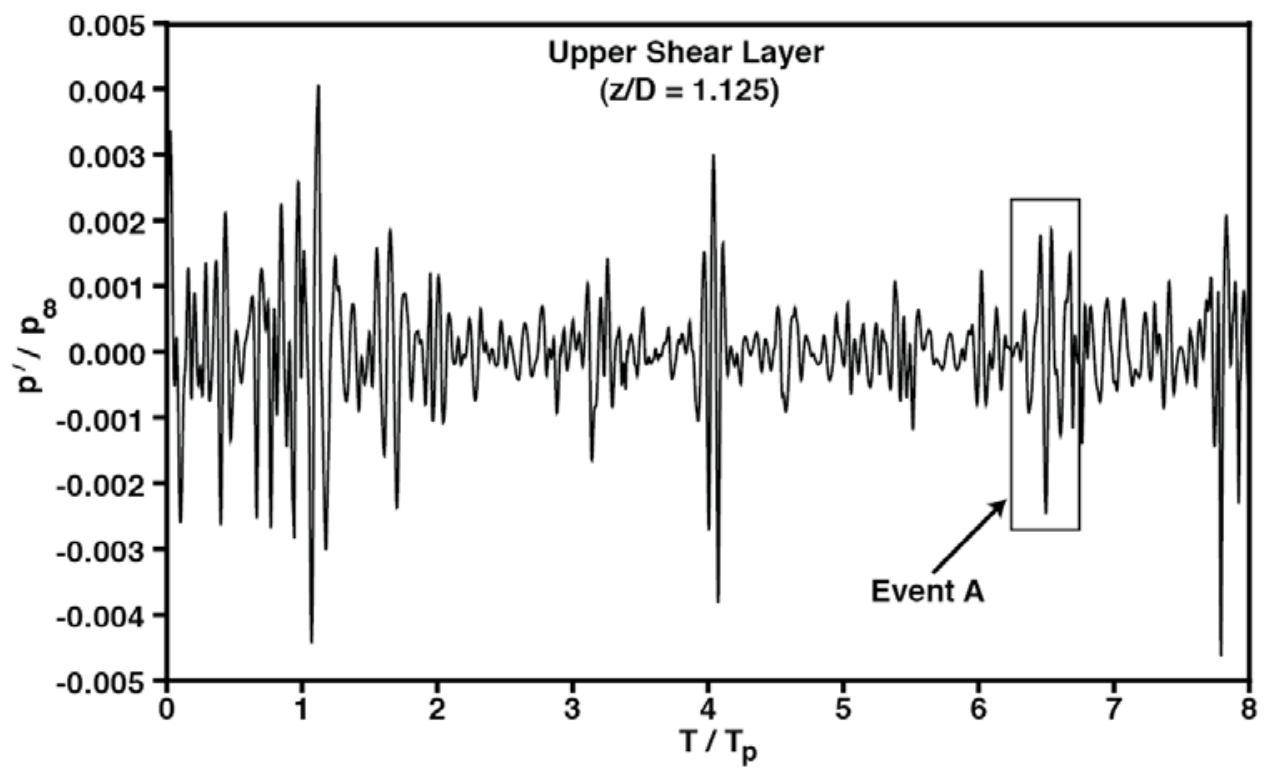

Figure 11. Fluctuating component of pressure obtained at $z / D=1.125$ in the upper shear layer (after application of high-pass filter as in Fig. 9). 
Figure 12 shows the computed pressure spectrum at the points marked ' $x$ ' in Figs. $4 \& 7$ (the two are averaged to obtain the plot in Fig. 12). The spectrum shows a sharp peak at shedding frequency and a broadband peak at an estimated value of about $\omega_{\mathrm{sl}} / \omega_{\mathrm{st}}=4.9$. Even with a sample size that is 100 shedding periods long, the computed spectrum shows slight oscillations. The spectrum was filtered using a three-point filter to obtain a smooth curve from which the location of the second peak was easily determined. The two spectra are nearly identical. Obtaining the dependence of $\omega_{\mathrm{sl}} / \omega_{\mathrm{st}}$ on $\operatorname{Re}_{\mathrm{D}}$ will require more simulations such as the present one for different values of $\mathrm{Re}_{\mathrm{D}}$.

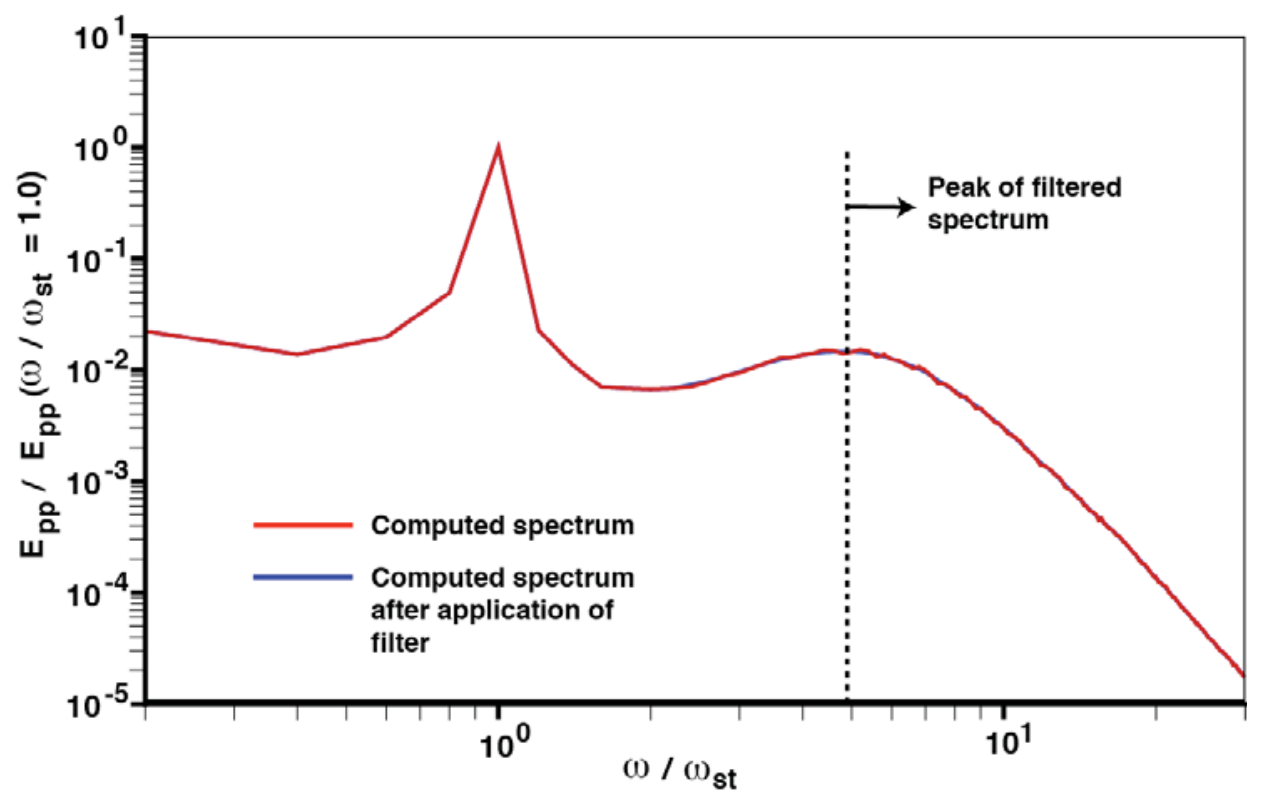

Figure 12. Spectrum of fluctuating pressure obtained within the detached shear layers.

\section{Rib Vortex induced Reverse Flow}

Here we investigate another phenomenon that is prevalent in the very near wake of the flat plate, reverse flow that is induced by powerful rib vortices. These vortices are formed when the shed vortices have high peak vorticity levels and are in close proximity to each other thus creating high rates of strain in a region in between. Figure 13a shows contours of instantaneous pressure in the wake center-plane (y/D $=0.0$ ). The region shown extends to $x / D=2.5$ and comprises the entire spanwise extent of the computational domain. The arrows point to three pressure minima that lie in the centers of rib vortices that at $y / D=0.0$ are closely oriented with the $y$ direction. There are other weaker minima in this figure that also correspond to rib vortices. Animations of pressure show these minima being first formed and subsequently convected from very near the trailing edge, downstream. Figure $13 \mathrm{~b}$ shows contours of instantaneous cross-stream vorticity at the same instant in time. The vortices A, B \& C are seen here as well. Figure $13 \mathrm{~b}$ indicates that a significant portion of the vorticity in the associated vortex may be aligned with the $y$ direction at $y / D=0.0$. This will be substantiated shortly. Figure $13 \mathrm{c}$ shows contours of negative streamwise velocity, $u$. As expected $u$ is for the most part negative in the near base region.

The remarkable finding here is that there are regions of negative $u$ associated with the vortices $A$, B \& C (and also with some of the other weaker vortices in Figs. 13a \& 13b). These regions are separated from the main body of reverse flow. In other words the rib vortices are sufficiently powerful to induce reverse flow in a region where the main flow is in the positive $\mathrm{x}$ direction (cross-stream vortices induce $u$ and $w$ velocity fields). Thus we have isolated regions of reverse flow (unattached to any surface) being convected downstream. 


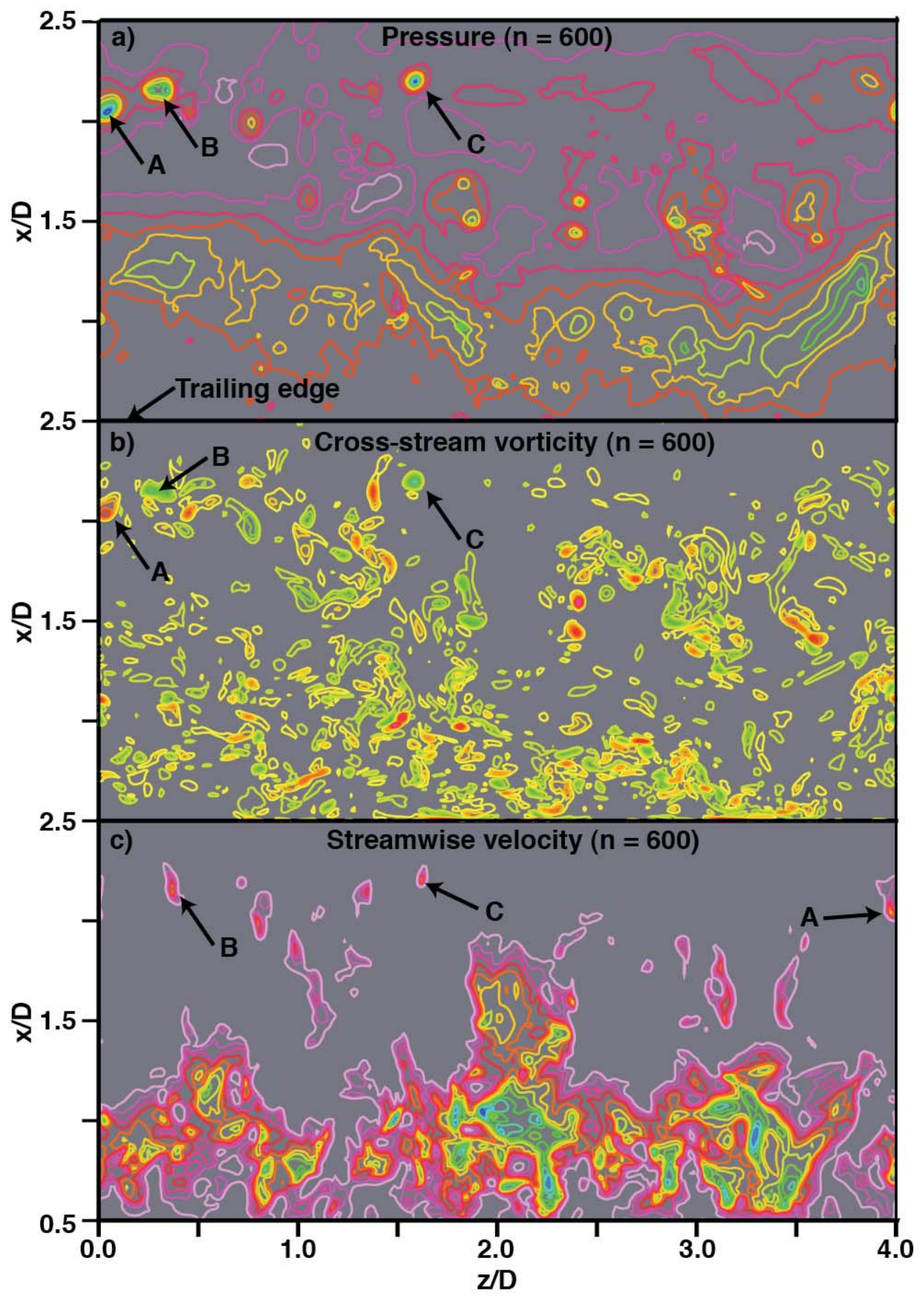

Figure 13. Wake centerplane in the very near wake $(x / D<2.5)$ showing instantaneous contours of a) pressure, b) cross-stream vorticity and c) negative streamwise velocity.

Figure 14a shows contours of instantaneous negative $u$ in an $(x, y)$ plane at the same instant in time as Fig. 13. This plane cuts through vortex $A$. The isolated patch of reverse flow (detached from the main region of reverse flow) is marked as 'rib vortex'. Figures $14 b-14 c$, also at the same instant in time, are of contours of instantaneous pressure, streamwise vorticity and cross-stream vorticity, and clearly show the associated rib vortex in the braid between two successive shed vortices. Only one of the shed vortices (large negative vortex above the rib vortex) is visible in this figure; the next one is downstream of $x / D=2.85$. The streamwise vorticity associated with this rib vortex at $y / D=0.0$ is relatively small compared to cross-stream vorticity, that is, the rib vortex is largely oriented with the $y$ direction. Its 
footprint on the center-plane is seen in Fig. 13a \& 13b. Plots of contours of negative $u$ at instants in time prior to that of Figs. $13 \& 14$ show the formation and evolution of the detached region of separated flow.

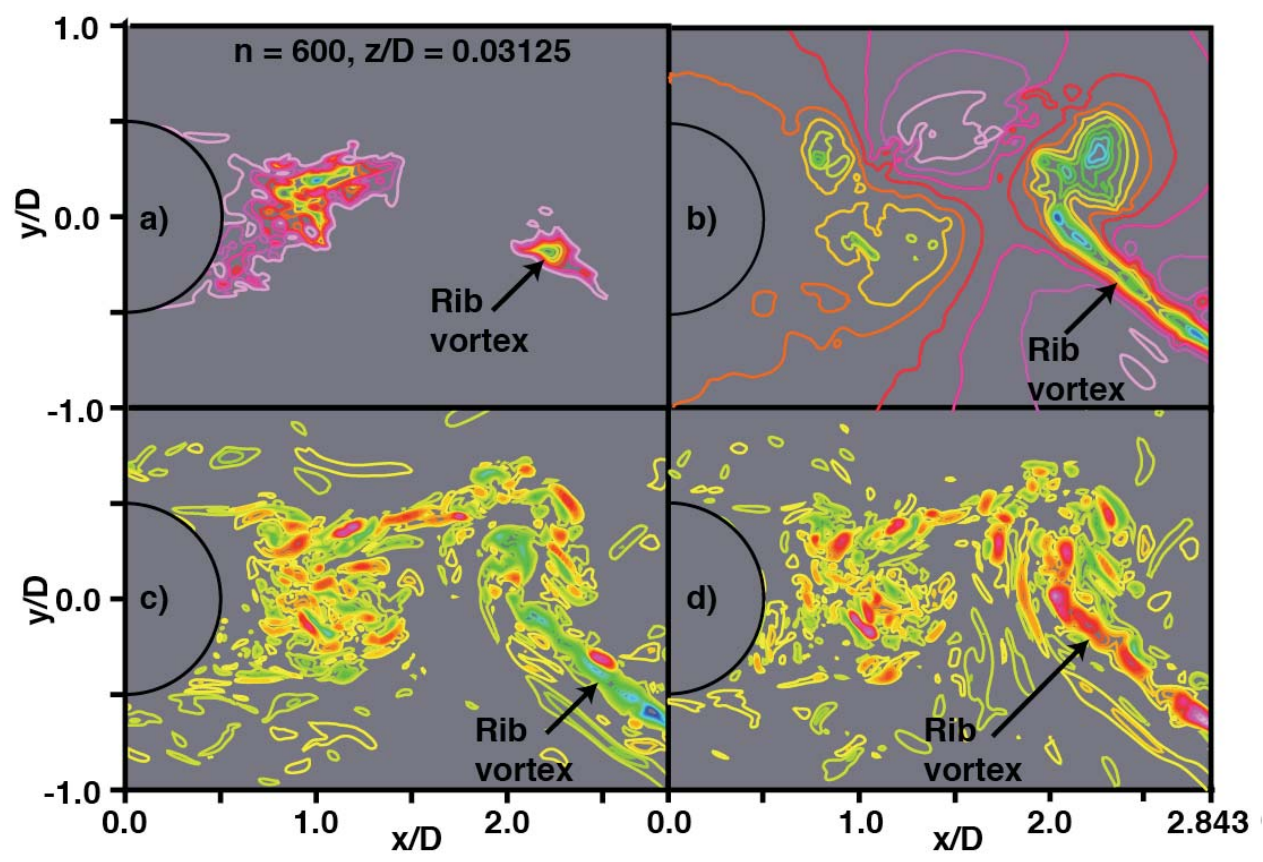

Figure 14. Contours in an ( $x, y)$ plane of instantaneous a) negative streamwise velocity, $b)$ pressure, c) streamwise vorticity and d) cross-stream vorticity.

The isolated regions of separation generally become smaller and weaker as they convect even further downstream $(x / D>3.0)$ and eventually disappear. Some of the weakening of the reverse flow may simply be due to the increasing streamwise velocity of the surrounding flow with increasing $x$, or, a reorientation of the rib vortex so that it is not as closely aligned with the $y$ direction. These regions of localized separation have been observed as far downstream as $x / D=4.0$ where the mean velocity is a significant fraction of the freestream velocity. We note that although the localized region of reverse flow associated with vortex $A$ has been attributed to the rib vortex, this region is located just below the negative shed vortex. The shed vortex reduces streamwise velocity in this region thus creating conditions that are favorable for reverse flow. The rib vortex then causes flow reversal. In all the cases observed so far, localized flow reversal has been accompanied by rib vortices. This is the reason we view the rib vortices as the causative agent for the phenomenon of localized reverse flow.

\section{Phase-Averaged Statistics}

The fluctuating density, pressure and velocity components in the flow field are assumed to contain a mean component, a periodic component at the shedding frequency $\omega_{\text {st }}$ (and harmonics thereof), and a random turbulent component. Thus a flow variable $\mathbf{q}$ can be written $\mathrm{ss}^{7}$

$$
\mathrm{q}=\overline{\mathrm{q}}+q^{\prime}+\mathrm{q}^{\prime \prime}
$$

where $\overline{\mathrm{q}}$ is the mean value, and $\mathrm{q}^{\prime}$ and $\mathrm{q}^{\prime \prime}$ are the random and periodic fluctuating components, respectively. The phase-averaged value $\langle q\rangle$ (average at constant phase) is given by

$$
<\mathrm{q}(\phi)>=\overline{\mathrm{q}}+\mathrm{q}^{\prime \prime}(\phi)
$$


where $\phi$ is the phase. The computed data were averaged in the spanwise direction to obtain the phaseaveraged data since the flow is assumed to be homogeneous in this direction. The method used to compute phase-averages is discussed in Ref. 1. Here, as in Ref. 1 , the phase $\phi=0$ corresponds to instants in time when the centers of the upper shed vortex reach the location $x / D=5.5$.

Contours of the phase-averaged spanwise vorticity, $\left\langle\Omega_{z}\right\rangle$, at one particular phase $\phi=1 / 6$, are shown in Fig. $15 a$ (very near wake, $x / D \leq 3.0$ ). Distributions of intensities, shear stress and various budget terms at this particular phase are representative (in a qualitative sense) of the same distributions obtained at other phases. Three distinct vortices (centers marked 1, 2 \& 3) are seen in Fig. 15a.

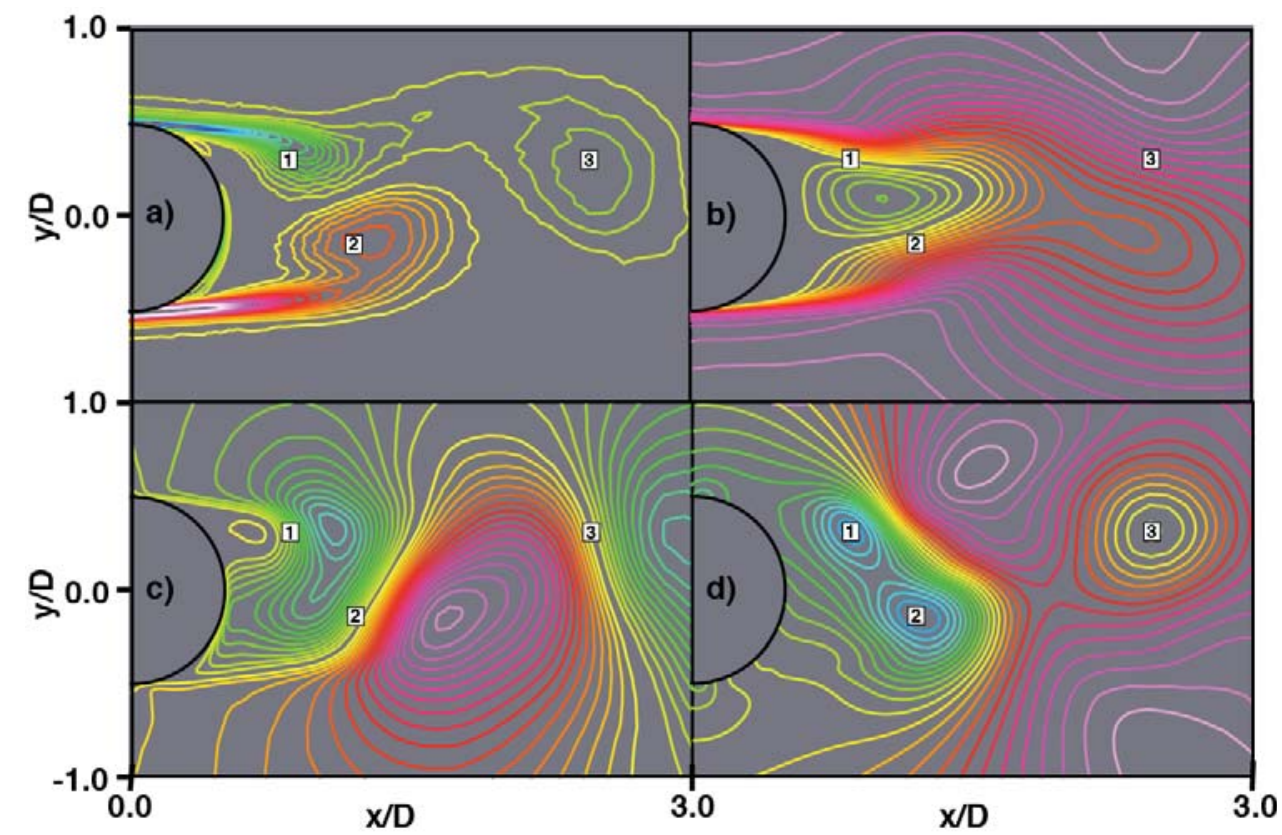

Figure 15. Phase-averaged a) spanwise vorticity, b) streamwise velocity, c) cross-stream velocity and d) pressure, $\phi=1 / 6$; in Figs. 15a, 15b \&15c red/magenta represent high/highest positive values, green/blue low/lowest negative values, yellow $\approx 0.0$.

Vortex 1 is still in the inception stage whereas vortices $2 \& 3$ are fairly well formed. The evolution of shed vortices with increasing phase (or time) can be discerned from these three vortices. Of interest is the growth in size and decrease in peak vorticity with increasing distance from the trailing edge. The ratio of peak vorticity obtained in vortices $2 \& 3$ is about 1.75 , which is substantial given that the streamwise distance between the two is only about 1.15D. Figure 15b shows phase-averaged contours of the streamwise velocity, $\langle u\rangle$. The contour line corresponding to $\langle u\rangle=0.0$ has been omitted in this figure to clearly demarcate the region of reverse flow (triangular in shape, extending to about $x / D=1.5$ ) from the rest of the region. Vortices $1 \& 2$ occur on either side of this region and both of them induce reverse flow therein, yielding a negative peak in $\langle u\rangle$. Figure $15 \mathrm{c}$ shows phase-averaged contours of the cross-stream velocity, $\langle v\rangle$. The contour $\langle v\rangle=0.0$ has been omitted to clearly demarcate regions of positive and negative $\langle v\rangle$. As in the case of $\langle u\rangle$ the peaks can be understood from the velocities induced by the three vortices. Figure 15d shows contours of phase-averaged pressure $\langle p\rangle$. As expected a pressure minimum is obtained at each of the vortex centers. Here and in the following figures in this subsection, phase-averaged vortex position is marked by the location of the corresponding pressure minimum.

Figures 16a, 16b, 16c \& 16d show the phase-averaged distributions of the random component of the streamwise $\left\langle u^{\prime} u^{\prime}\right\rangle$, cross-stream $\left\langle v^{\prime} v^{\prime}\right\rangle$, and spanwise $\left\langle w^{\prime} w^{\prime}\right\rangle$ normal intensities and shear stress $\left\langle u^{\prime} v^{\prime}\right\rangle$, respectively, at $\phi=1 / 6$. These distributions undergo considerable changes as they 
convect downstream. One of the objectives here is to compare the distribution of these stresses as obtained in the near and very near wakes. For this purpose we have included Fig. 17 (Fig. 6 from Ref. 1) which shows the distribution of the stresses at $\phi=0$ in the region $4.75<x / D<13.03$, where the distributions have attained their near-wake characteristics. The fact that Fig. 17 corresponds to a slightly earlier phase is not important (the features in Fig. 17 simply convect about 0.7D downstream and show small changes in shape and magnitude at $\phi=1 / 6)$. We now focus on some of the important features of the distributions in Figs. $16 \& 17$ and the commonalities/differences.

One difference between Figs. $16 \& 17$ is that the minimum/maximum values specified in Fig. 17 are about half as much as those in Fig. 16. However the subplots within each figure all have the same minimum/maximum values and increment between contours. The subplots in Figs. $16 \& 17$ provide a numerical value for a factor $f($ e.g. $f=4.0)$. This is the factor that the quantity in the subplot is multiplied by so that its minimum/maximum values are about the same as those in the other subplots. This operation ensures that the number of contours in each subplot is sufficient to depict features of interest.

Contours of $\left\langle u^{\prime} u^{\prime}\right\rangle$ in Fig. 17 show maxima both in the braids and near the vortex cores while contours of $\left\langle v^{\prime} v^{\prime}\right\rangle$ on the other hand show maxima slightly upstream of the cores and a saddle-point in each braid. These features and their origin are discussed at length in Ref. 1. The distributions of Fig. 16 (very near wake) are noticeably different from those of Fig. 17 (near wake). Figure 16a for example shows a braid like region that seems to extend between the upper two vortices (vortices 1 \& 3 ) instead of between vortices $2 \& 3$ as Fig. 17a would suggest. The braid in Fig. 16a contains a maximum in intensity. The maxima at the cores (seen in Fig. 17a) are not yet evident. The maximum near the core of vortex 2 is actually a precursor to the braid like region that resembles the upper one; it acquires this form at a later phase. Contours of $\left\langle w^{\prime} w^{\prime}\right\rangle$ (Fig. 16c) show only the upper braid like region and not the maxima near the cores as in Fig. 17c. On the other hand Fig. 16b $\left.\left(<v^{\prime} v^{\prime}\right\rangle\right)$, shows the maxima associated with the cores well defined early in the vortex formation process. The braids and saddle points can also be discerned in this figure. Contours of $\left\langle u^{\prime} v^{\prime}\right\rangle$ in Fig. 16d do resemble those of Fig. 17d (with peaks in the braids and near the cores). In general intensity and shear stress levels are two or more times larger in Fig. 16 than in Fig. 17 (choice of min/max values and color scheme).

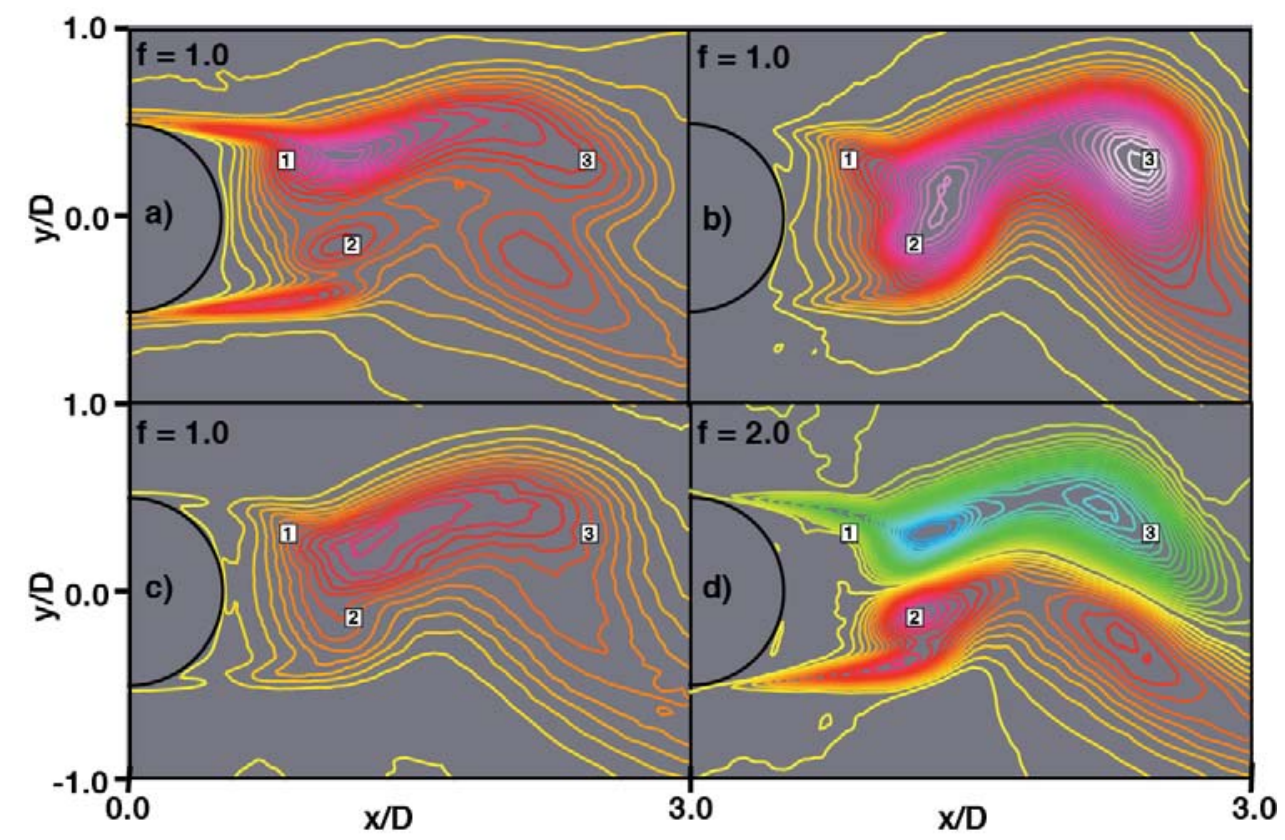

Figure 16. Contours of a) $\left\langle u^{\prime} u^{\prime}\right\rangle$, b) $\left\langle v^{\prime} v^{\prime}\right\rangle$, c) $\left\langle w^{\prime} w^{\prime}\right\rangle$ and d) $\left\langle u^{\prime} v^{\prime}\right\rangle$ at $(\phi=1 / 6, x / D<3.0)$; $\mathrm{red} /$ magenta represent high/highest positive values, green/blue low/lowest negative values, yellow $\approx 0.0$. 


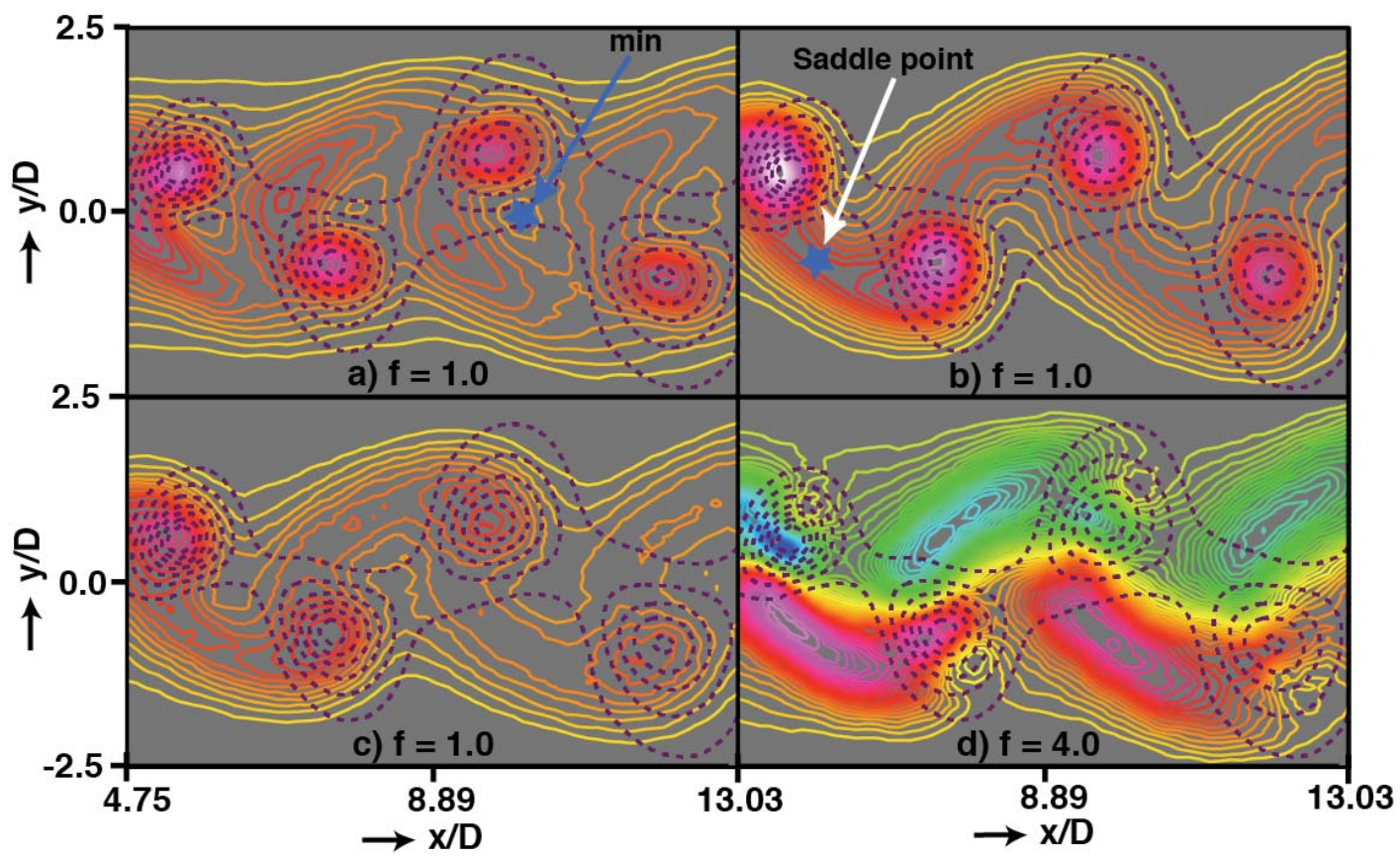

Figure 17. Contours of a) $\left\langle u^{\prime} u^{\prime}\right\rangle$, b) $\left\langle v^{\prime} v^{\prime}\right\rangle$, c) $\left\langle w^{\prime} w^{\prime}\right\rangle$ and d) $\left\langle u^{\prime} v^{\prime}\right\rangle(\phi=0)$; red/magenta represent high/highest positive values, green/blue low/lowest negative values, yellow $\approx 0.0$. Dashed lines are contours of phase-averaged pressure and show vortex centers (Figure 6 from Ref. 1).

Another major difference is that the detached shear layers and the flow adjacent to the trailing edge (see phase-averaged intensity/shear-stress contours in Fig. 16) do not have counterparts in Fig. 17.

Here as in Ref. 1, distributions of the production terms in the transport equations for the stresses are investigated to better understand the distribution of phase-averaged turbulent stresses obtained in the wake (Fig. 16). For the incompressible form of the transport equations we obtain ${ }^{1}$,

$$
P_{i j}=-\rho\left(<u_{j}^{\prime} u_{k}^{\prime}>\frac{\partial<u_{i}>}{\partial x_{k}}+<u_{i}^{\prime} u_{k}^{\prime}>\frac{\partial<u_{j}>}{\partial x_{k}}\right)
$$

where $\rho$ is the density, and $u_{i}(i=1,2,3)$ are the velocity components in the $\mathrm{x}, \mathrm{y} \& \mathrm{z}$ directions $\left(x_{i}\right)$.

Figure 18a shows contours of the turbulence production term $\left\langle P_{11}\right\rangle$ in the budget of $\left\langle u^{\prime} u^{\prime}\right\rangle$, given by,

$$
P_{11}=-2 \rho\left[\left\langle u^{\prime} u^{\prime}\right\rangle \frac{\partial<u>}{\partial \mathrm{x}}+\left\langle u^{\prime} v^{\prime}>\frac{\partial<u>}{\partial \mathrm{y}}\right]\right.
$$

The term $\left\langle u^{\prime} w^{\prime}\right\rangle \partial\langle u>/ \partial z$ ) does not appear in Eq. 3b (homogeneity in the $z$ direction). Figure 18a shows a high rate of turbulence production in the braid region between the upper two vortices that is consistent with the region of high $\left\langle u^{\prime} u^{\prime}\right\rangle$ in Fig. 16a. This is partly a result of the stretching of rib vortices that are present in this region (discussed later in the text). The high production rate of $\left\langle u^{\prime} u^{\prime}\right\rangle$ in the braid can also be surmised from Eq. 3b; the y derivative of $\langle u\rangle$ is substantially (positive) in this region, and the shear stress is negative (see Fig. 15b \& 16d). At the same time the $x$ derivative of $\langle u\rangle$ in this region is relatively small. Hence it is the second term in Eq. $3 \mathrm{~b}$ that dominates thus resulting in production. An area of turbulence consumption just below this braid region in seen in Fig. 18a. Figure 15b shows the $y$ derivative of $\langle u\rangle$ is nearly zero along the centerline in the region of consumption. Hence it is the first term in Eq. $3 \mathrm{~b}$ that is dominant. Given that the $\mathrm{x}$ derivative of $\langle u>$ is positive in this region and since the streamwise intensity is always positive, we have turbulence consumption. 


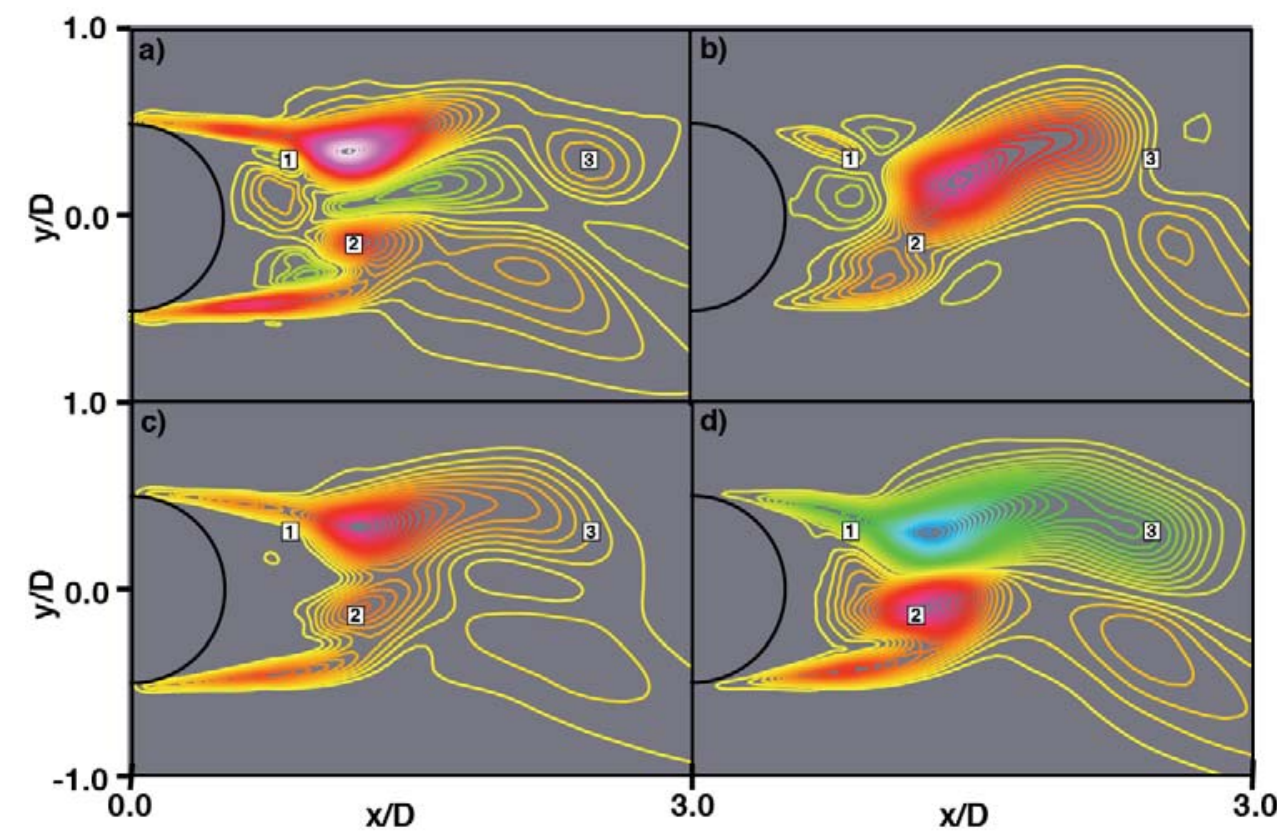

Figure 18. Phase-averaged production terms a) $P_{11}$, b) $P_{22}$, c) $0.5\left(P_{11}+P_{22}\right) \&$ d) $P_{12}(\phi=1 / 6)$; $\mathrm{red} / \mathrm{magenta}$ represent high/highest positive values, green/blue low/lowest negative values, yellow $\approx 0.0$.

Figure $18 \mathrm{~b}$ shows contours of the production term $P_{22}$ in the budget of $\left\langle v^{\prime} v^{\prime}\right\rangle$, given by,

$$
P_{22}=-2 \rho\left[\left\langle u^{\prime} v^{\prime}\right\rangle \frac{\partial\langle v\rangle}{\partial x}+\left\langle v^{\prime} v^{\prime}\right\rangle \frac{\partial<v>}{\partial y}\right]
$$

The entire braid region here shows positive values for the production term; this is consistent with the high levels of intensity in this region seen in Fig. 16b. Figure 18c shows the distribution of the production term in the phase-averaged turbulent kinetic energy (TKE)

$$
P_{T K E}=-\left[\left\langle u^{\prime} u^{\prime}\right\rangle \frac{\partial\langle u\rangle}{\partial x}+\left\langle u^{\prime} v^{\prime}\right\rangle\left(\frac{\partial\langle v\rangle}{\partial x}+\frac{\partial\langle u\rangle}{\partial y}\right)+\left\langle v^{\prime} v^{\prime}\right\rangle \frac{\partial\langle v\rangle}{\partial y}\right]
$$

Regions of negative production are negligible in this combined production term, demonstrating that the positive production rate of $\left\langle u^{\prime} u^{\prime}\right\rangle$ more than compensates the negative rate obtained for $\left\langle v^{\prime} v^{\prime}\right\rangle$ adjacent to vortex cores $1 \& 2$. Similarly the negative production rate of $\left\langle u^{\prime} u^{\prime}\right\rangle$ discussed above is compensated by the positive production rate of $\left\langle v^{\prime} v^{\prime}\right\rangle$. The production rate for $\left\langle w^{\prime} w^{\prime}\right\rangle$ is zero because $\langle w\rangle$ is zero everywhere. It is probably the velocity pressure gradient term that contributes to the sustenance of $\left\langle w^{\prime} w^{\prime}\right\rangle$. Clearly the stretching of rib vortices (amplification of streamwise and crossstream vorticity) in the braid contributes significantly to the creation of $w^{\prime}$. Figure $18 d$ shows contours of the production term in the transport equation for $\left\langle u^{\prime} v^{\prime}\right\rangle$ given by

$$
P_{12}=-\rho\left[\left\langle u^{\prime} u^{\prime}\right\rangle \frac{\partial\langle v\rangle}{\partial x}+\left\langle v^{\prime} v^{\prime}\right\rangle \frac{\partial\langle u\rangle}{\partial y}\right]
$$

This term is for the most part negative above the wake centerline and positive below the centerline and resembles the distribution obtained for $\left\langle u^{\prime} v^{\prime}\right\rangle$ in Fig. $16 \mathrm{~d}$. We note that the various features seen in Figure 18 can be explained via the distributions in Figs. 15 \& 16 and Eq. 3a (just as in the case of turbulence consumption in Fig. 18a); this exercise is left to the interested reader.

Figures 16 - 18 clearly indicate that the dynamics of the very near wake are substantially different from those of the near wake; information that needs to be taken into account both in attempts to 
understand the basic physics in the two regions and in turbulence modeling. The reverse flow near the trailing edge and the detached shear layers are important contributors to the differences in the distributions of turbulence intensity, shear stress, production, dissipation and transport in the two regions.

Figure 19 shows surfaces of constant vorticity magnitude (instantaneous), streamwise and crossstream components only $\Omega=\left(\Omega_{\mathrm{x}}{ }^{2}+\Omega_{\mathrm{y}}{ }^{2}\right)^{1 / 2}$. Figure 19 a shows a perspective view in the region $0<\mathrm{x} / \mathrm{D}<$ 2.83 and Fig. 19b the corresponding top view. The time instant was chosen so that the locations of three consecutive vortices (labeled v1, v2 \& v3) are nearly the same as the locations of the phase-averaged vortices marked 1, 2 \& 3 in Fig. 18a. Numerous rib vortices are evident in both Figs. 19a \& 19b between v1 \& v2 and between v2 \& v3. These rib vortices contribute significantly to both the intensity levels and shear stress (via induced velocities) and also to turbulence production. As discussed in in Ref. 1, production is achieved by the stretching of rib vortices via the phase-averaged strain rate in the braids and by turbulent stretching (fluctuating strain rate).
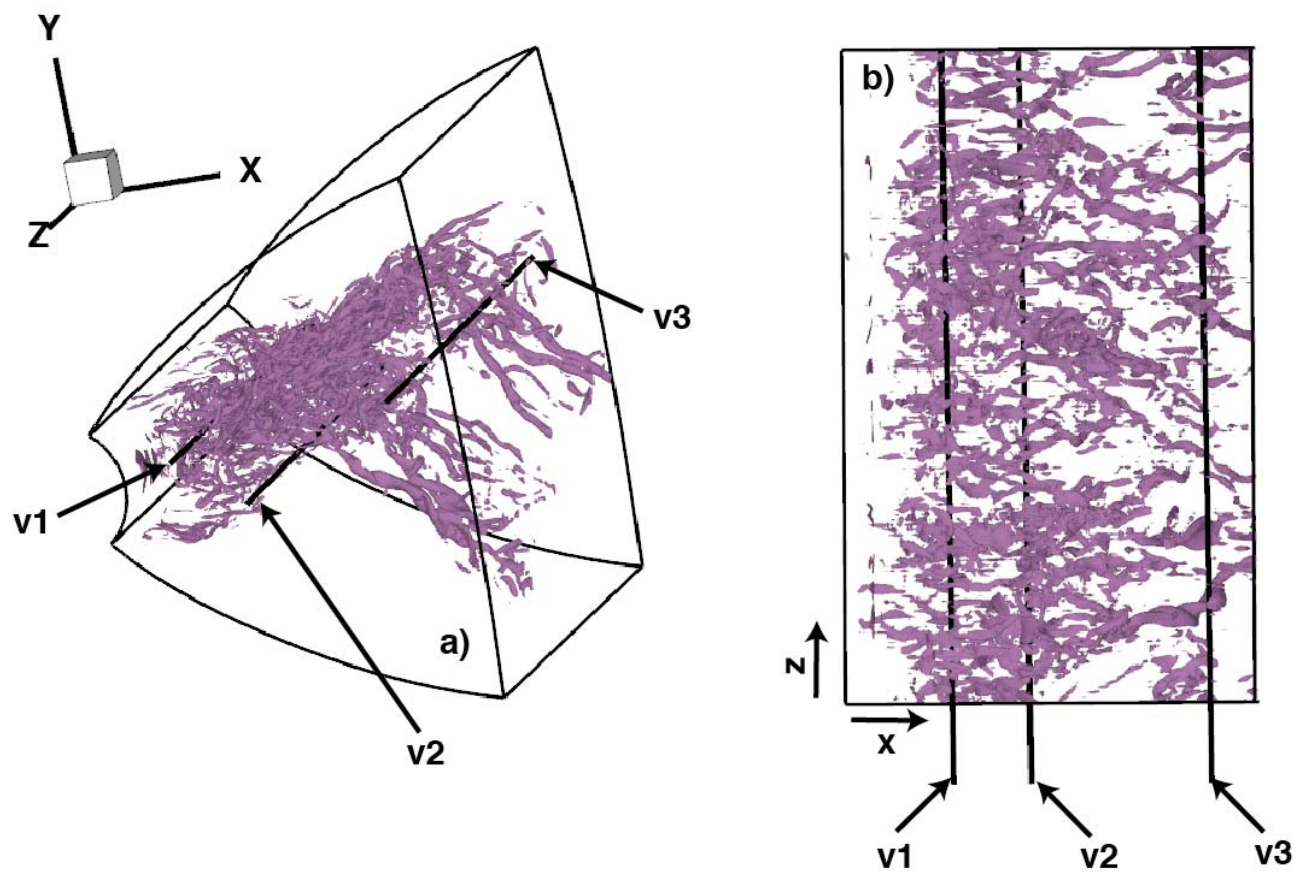

Figure 19. Surfaces of constant vorticity magnitude, streamwise and cross-stream components only $\Omega=$ $\left(\Omega_{\mathrm{x}}^{2}+\Omega_{\mathrm{y}}{ }^{2}\right)^{1 / 2}$ (maximum value of $\mathrm{x} / \mathrm{D}=2.83$ ), a) perspective view, b) top view.

\section{SUMMARY}

The very near wake of a thick flat plate with a circular trailing edge, with pronounced shedding of vortices, is investigated here via DNS. The emphasis of the present study is on the stability of the detached shear layers, rib-vortex induced separation, and phase-averaged distributions of the random component of normal intensities and shear stress and the production term in the corresponding budgets.

As in the case of the cylinder with laminar separating boundary layers, we have shear layer instability followed by the formation of shear layer vortices that have a profound impact on the structure of the shear layer and the formation of the shed vortices. However, unlike the cylinder cases, here only a small fraction of the separating turbulent boundary layer participates in both the instability process as well the initial formation of the shed vortices. The shed vortices themselves were to found to comprise shear layer vortices from the upper and lower shear layers, recirculation region vortices and segments of the shear layers that wrap around a central core. While both positive and negative vortices are found in the shed vortices, net vorticity is of the same sign as the vorticity in the generating shear layer. 
Here as in Ref. 2 (cylinder case), periods of shear layer instability correlated well with the interaction of the shear layer with recirculation region vortices, and quiescent periods showed little or no interaction between the two. This is a strong indicator that the interaction is a primary contributor to initiation of the instability. The role of the interaction between the shear layer and disturbances in the outer portion of the separated boundary layer in destabilizing this layer is yet to be investigated.

Spectra of the time-varying velocity and pressure within the shear layers at $x / D=0.5$ were obtained. Unlike the cylinder case ${ }^{2}$ the spectrum of streamwise velocity did not show a broadband peak because of the powerful velocity fluctuations that are already present in the shear layer at its inception (turbulent boundary layer). The pressure signal on the other hand did show a clear broadband peak with the characteristic, associated shear layer frequency. The ratio of this frequency to the shed frequency was estimated to be about 4.9 for the flat plate geometry and flow parameters chosen. Obtaining the relationship between this ratio and $\mathrm{Re}_{\mathrm{D}}$ is of considerable interest. Additional simulations of the type performed here for different values of $\mathrm{Re}_{\mathrm{D}}$ are required to establish this relationship.

The distribution of phase-averaged pressure, velocity components, vorticity, normal intensity \& shear stress (random fluctuating component) and the corresponding production terms in the budgets are examined here at one phase. The vorticity contours show a rapid decrease in peak vorticity values in the shed vortices with increasing $x$. The intensity contours show a braid like region still in its infancy with high levels of intensity and shear stress. Contours of instantaneous vorticity magnitude show numerous rib vortices in this region thus explaining the high levels of intensity and shear stress. Although this particular feature is seen downstream in the near wake as well (albeit more developed with the braid occurring between successive vortices of opposite sign), there are other features that are not common to the very near and near wakes. Some of these differences can be attributed to the presence of the reverse flow region in the very near wake that profoundly alters the velocity field and thus the production of turbulence and, others to the presence of the detached shear layers. Distributions of phase-averaged turbulence dissipation and transport also show noticeable differences in these two regions; differences that need to be accounted for in efforts to understand the basic physics and to model turbulence in the shedding wake.

\section{REFERENCES}

1. Rai, M. M., "Flow Physics in the Turbulent Near Wake of a Flat Plate," Journal of Fluid Mechanics, Volume 724, 2013, pp. 704-733.

2. Rai, M. M., "A Computational Investigation of the Instability of the Detached Shear Layers in the Wake of a Circular Cylinder," Journal of Fluid Mechanics, Volume 659, 2010, pp. 375-404.

3. Rai, M. M., "Direct Numerical Simulation of Transitional and Turbulent Flow on a Turbine Airfoil," $\underline{\text { AlAA }}$ Journal of Propulsion and Power, Volume 26, No. 3, May-June 2010, pp. 587-600.

4. Rai, M. M., "Towards Direct Numerical Simulations of Turbulent Wakes," AIAA Paper No. 2008-0544, $46^{\text {th }}$ AIAA Aerospace Sciences Meeting, Reno, Nevada, January 7-10, 2008.

5. Karlson, R. I. \& Johansson, T. G. 1988 "LDV Measurements of higher-order moments of velocity fluctuations in a turbulent boundary layer," Laser Anemometry in Fluid Mechanics, Ladoan-Instituto Superior Tecnico, Portugal, 1988.

6. Bloor, M. S., "The Transition to Turbulence in the Wake of a Circular Cylinder," Journal of Fluid Mechanics, Volume 19, 1964, pp. 290-304.

7. Reynolds, W. C. and Hussain, A. K. M. F., "The Mechanics of an Organized Wave in Turbulent Shear Flow. Part 3. Theoretical Models and Comparisons with Experiments," Journal of Fluid Mechanics, Volume 54, 1972, pp. 263-288. 